\title{
A model for characterization of a vortex pair formed by shock passage over a light-gas inhomogeneity
}

\author{
By JOSEPH YANG ${ }^{1}$, TOSHI KUBOTA ${ }^{2}$ \\ AND EDWARD E. ZUKOSKI \\ ${ }^{1}$ Department of Mechanical Engineering, California Institute of Technology, \\ Pasadena, CA 91125, USA \\ ${ }^{2}$ Department of Aeronautics, California Institute of Technology, Pasadena, CA 91125, USA
}

(Received 11 October 1991 and in revised form 15 June 1993)

This work investigates the two-dimensional flow of a shock wave over a circular lightgas inhomogeneity in a channel with finite width. The pressure gradient from the shock wave interacts with the density gradient at the edge of the inhomogeneity to deposit vorticity around the perimeter, and the structure rolls up into a pair of counter-rotating vortices. The aim of this study is to develop an understanding of the scaling laws for the flow field produced by this interaction at times long after the passage of the shock across the inhomogeneity. Numerical simulations are performed for various initial conditions and the results are used to guide the development of relatively simple algebraic models that characterize the dynamics of the vortex pair, and that allow extrapolation of the numerical results to conditions more nearly of interest in practical situations. The models are not derived directly from the equations of motion but depend on these equations and on intuition guided by the numerical results. Agreement between simulations and models is generally good except for a vortex-spacing model which is less satisfactory.

A practical application of this shock-induced vortical flow is rapid and efficient mixing of fuel and oxidizer in a SCRAMJET combustion chamber. One possible injector design uses the interaction of an oblique shock wave with a jet of light fuel to generate vorticity which stirs and mixes the two fluids and lifts the burning jet away from the combustor wall. Marble proposed an analogy between this three-dimensional steady flow and the two-dimensional unsteady problem of the present investigation. Comparison is made between closely corresponding three-dimensional steady and twodimensional unsteady flows, and a mathematical description of Marble's analogy is proposed.

\section{Introduction}

The motivation for the study of shock-induced vortical flows is combustion in a supersonic combustion ramjet (SCRAMJET), where supersonic air flow into the combustion chamber limits the residence time to a few milliseconds. This imposes a severe requirement for rapid and efficient mixing of fuel and oxidizer. In addition, heat transfer considerations dictate that combustion occur relatively far from the combustion chamber wall.

F. E. Marble (1985, personal communication) first proposed the mechanism of shock-induced vorticity generation for SCRAMJET combustion. He argued that the 


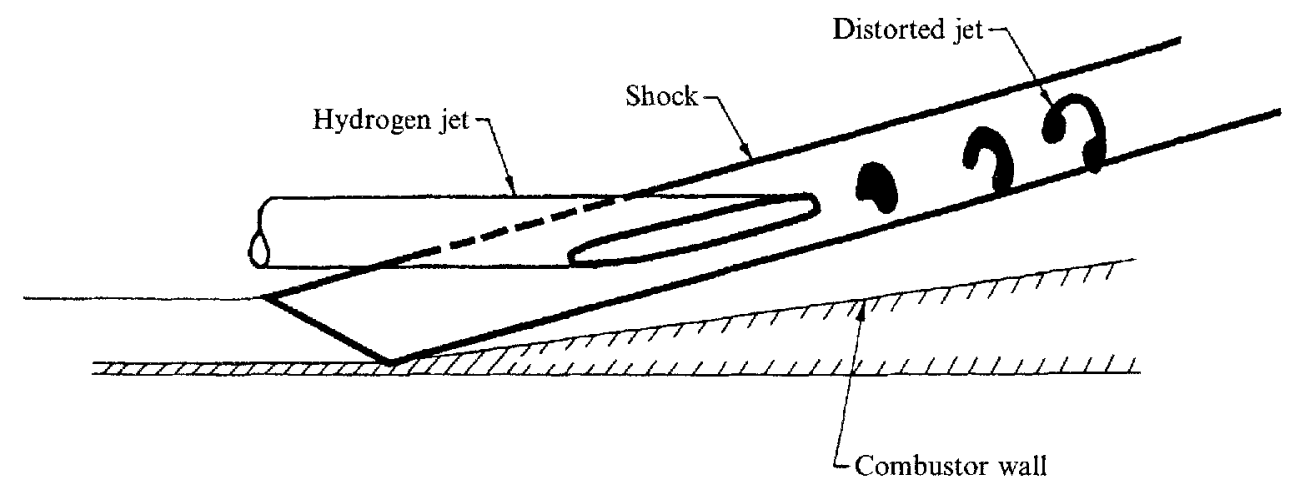

FIGURE 1. Schematic of a three-dimensional steady shock-induced vortical flow (reproduced from Marble et al. 1990, p. 7).

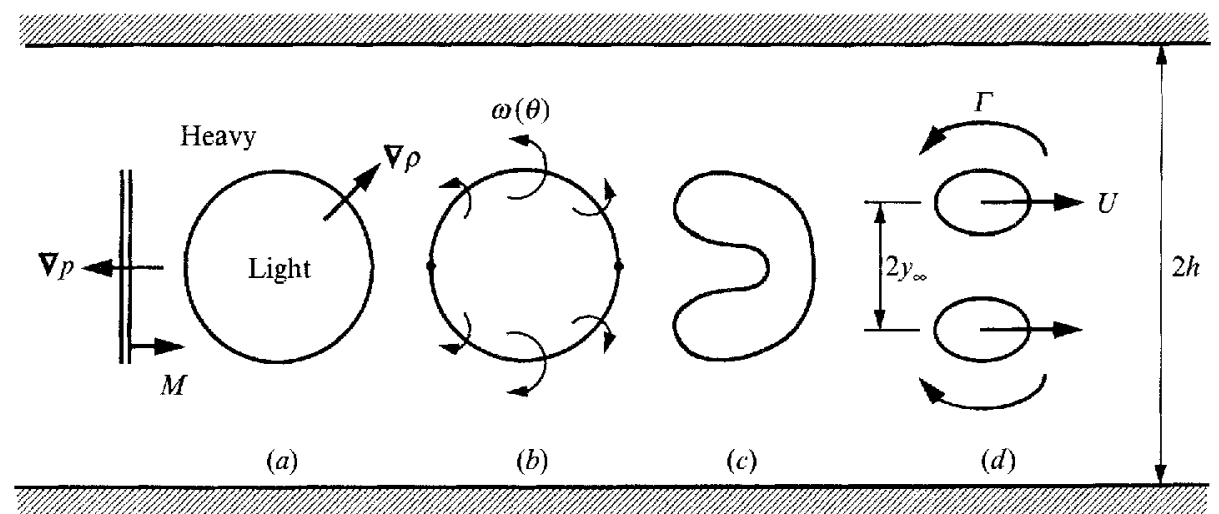

FIGURE 2. Schematic of a two-dimensional unsteady shock-induced vortical flow: (a) $t=0^{-}$, (b) vorticity distribution at $t=0^{+},(c)$ roll up, $(d)$ steady-state vortex pair.

interaction of a shock wave with a jet of light gas surrounded by an ambient heavy gas would generate vorticity around the perimeter of the jet. This vorticity would then cause the jet to roll up at its front and rear edges, stirring and mixing the light jet gas with the ambient heavy gas (figure 1). In addition, circulation of the vortices would bring the trajectory of the burning jet up and away from the combustor wall. Description of the mixing and trajectory requires a kinematical and dynamical understanding of the flow, respectively. The authors considered the kinematics of shock-induced vortical flows in a previous paper (Yang, Kubota \& Zukoski 1993); the present work addresses their dynamics.

Marble argued that the three-dimensional steady flow in an actual injector design should be analogous to a two-dimensional unsteady flow, which is more conveniently studied either experimentally or computationally. In the three-dimensional flow, the shock wave passes vertically upward through the jet as it moves downstream. In the analogous two-dimensional flow, a normal shock wave propagates through a planar region of light gas and causes the gas to roll up at its upper and lower edges. This is illustrated in figure 2, which shows the time evolution of the two-dimensional structure. Spatial development in the three-dimensional flow corresponds to temporal development in the two-dimensional flow.

Study of this shock-induced vortical flow predates its proposed application to 
supersonic combustion. It was first investigated by Rudinger \& Somers (1960) and more recently by Haas (1984), Haas \& Sturtevant (1987), Picone \& Boris (1988), Marble, Hendricks \& Zukoski (1987), Marble et al. (1990), Hendricks \& Marble (1991) and Jacobs (1992). The studies all demonstrated the development of qualitatively similar vortical structures, but with unexplained quantitative discrepancies (e.g. differences in the size, spacing, and velocity of vortical structures formed from similar initial conditions).

The present work is an analytical and computational investigation of the shockinduced vortical flow in the two-dimensional unsteady case. The flow is simulated numerically, establishing a qualitative understanding of the important flow features, as well as quantifying the behaviour over a range of flow parameters. We consider only superficially the evolution of the vorticity field of the early-time, developing flow. Instead, we focus on the behaviour of the vortex pair at times long compared with the passage of the shock over the inhomogeneity. We develop algebraic models for the strength, velocity, spacing, and time of development of the vortex pair, and compare them against the results of the computations. These models correlate and summarize the numerical data, and also provide a tool for interpolation/extrapolation to situations within the range of, but not explicitly computed among, the finite number of flow parameters considered here. Finally, the analogy between two-dimensional unsteady flows and the corresponding three-dimensional steady flows is discussed both qualitatively and quantitatively.

\section{The canonical problem: interaction of a shock wave with a single circular jet}

Consider the interaction of a shock wave in air with a single circular region of lowerdensity gas, as shown in figure 2(a). This flow could represent, for example, a slice through a jet perpendicular to the plane of the paper. According to the inviscid vorticity equation,

$$
\frac{\mathrm{D}}{\mathrm{D} t}\left(\frac{\omega}{\rho}\right)=\frac{1}{\rho}(\omega \cdot \nabla u)+\frac{1}{\rho^{3}}(\nabla \rho \times \nabla p) .
$$

This can be rewritten as

$$
\frac{\mathrm{D} \omega}{\mathrm{D} t}=\frac{1}{\rho^{2}}(\nabla \rho \times \nabla p)+\ldots,
$$

giving baroclinic generation of vorticity proportional to the cross-product of the density and pressure gradients.

Passage of the shock wave deposits vorticity around the circumference of the jet, as shown in figure $2(b)$. The density gradient, at the circumference of the jet, is everywhere radially outward. The pressure gradient, across the shock, points upstream. Where these gradients are perpendicular, at the top and bottom of the jet, vorticity generation is maximum. Where they are parallel, at the upstream and downstream ends of the jet, vorticity generation is zero. At other circumferential locations, vorticity generation is intermediate.

The deposited vorticity will initially cause the jet to roll up into a kidney-shaped structure, as shown in figure $2(c)$. As time goes on, the vorticity will coalesce, and the structure will evolve towards a vortex pair with finite core size, as shown in figure $2(d)$. 


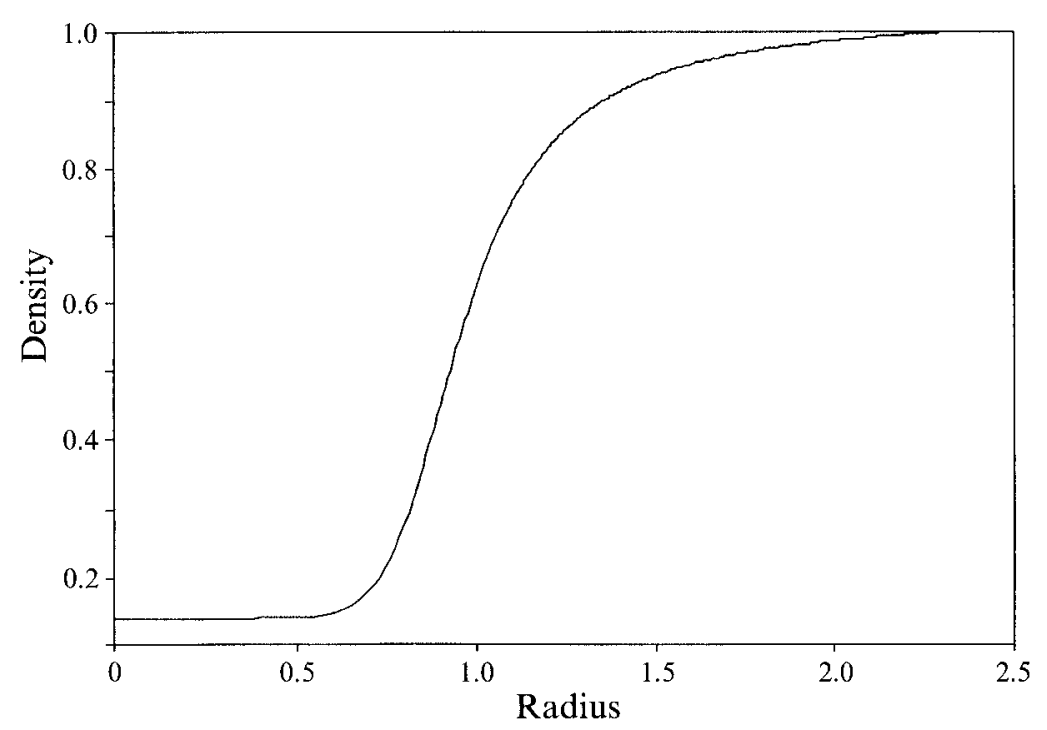

FIGURE 3. The laminar jet mixing layer used to initialize the computations.

\section{Governing equations and computational technique}

Physical variables are non-dimensionalized as follows: lengths are scaled by the initial radius: $\bar{x}=x / R_{0}$; velocities are scaled by the speed of sound ahead of the shock: $\bar{u}=u / c_{1}$; times are scaled by $R_{0} / c_{1}: \bar{t}=t c_{1} / R_{0}$; densities are scaled by the density ahead of the shock: $\bar{\rho}=\rho / \rho_{1}$; pressures are scaled by the pressure ahead of the shock: $\bar{p}=p / p_{1}$.

The flow is governed by mass, momentum, energy, and species equations for an ideal gas, neglecting diffusion and viscosity. These equations are solved using LCPFCT (Oran 1991), a recent version of SHASTA (Boris \& Book 1973), which is an explicit Eulerian finite-difference flux-corrected-transport (FCT) algorithm. The usual difficulty in finite-difference computations is that the numerical diffusion required for stability often overwhelms the actual physical diffusion. Thus it is difficult to capture strong gradients, such as shock waves, without smearing out fine details of the flow. LCPFCT features an antidiffusive correction stage that locally removes the numerical diffusion in excess of either the physically correct diffusion or that required for stability. Where stability is not threatened, the algorithm retains $0.1 \%$ of the original numerical diffusion, a level chosen to match the diffusion in a real flow $\left(\approx 8 \times 10^{-5}\right.$, in nondimensional units, for the experimental conditions of Jacobs 1992).

Initial conditions for the computations were chosen to match the conditions present in the experiments of Jacobs, who studied the flow produced when a plane shock wave impinged on a laminar jet of helium injected into air at ambient pressure. The density gradient at the edge of the light-gas cylinder was obtained using a similarity solution for the axisymmetric mixing layer at the edge of an axisymmetric jet (figure 3), at a plane 4.8 diameters downstream of the nozzle. This corresponds to one of the conditions used by Jacobs in his experiments.

The initial condition is integrated forward in time, with a timestep determined by a Courant condition. The $x$ - and $y$-integrations are treated using the technique of operator splitting. The channel is a rectangular region with a typical height of 8.0 and a length of 10.0. For the computations discussed here, the cylinder of light gas is placed on the centreline of the duct and consequently, the computational domain can be 
restricted to the upper half of the duct by symmetry arguments. The initial radius of the cylinder of light gas is 1.00 and the cell size for the computations is 0.05 or onetwentieth of the cylinder radius. During the computation, the rectangular domain is moved in time to track the developing vortex pair. The inflow and outflow boundaries specify zero-gradient flow and the upper and lower boundaries are perfectly reflecting.

Numerical resolution affects the results through shock wave thickness and numerical diffusion. The adequacy of the 0.05 grid spacing was checked in the worst case, $M=2.0$ and light/heavy gas density ratio 0.138 , by comparing results among computations with cell sizes of $0.10,0.05$, and 0.025 . Changes of as much as $12 \%$ in key parameters were observed when the cell size was reduced from 0.10 to 0.05 . However, when the cell size was reduced from 0.05 to 0.025 , there was excellent quantitative agreement between the two results. For example, the circulation and velocity of the vortex pair changed less than $3 \%$, and no change was observed in either the spacing of the vortex pair or the normalized velocity, i.e. the velocity times the spacing divided by the circulation. The reduction in cell size also provided an indirect check on the effect of numerical approximation to shock structure.

Because the CPU time required to carry out the computations is increased by a factor of eight for each factor-of-two reduction in cell size, the 0.05 spacing appears to be a reasonable compromise between cost and accuracy for this study of the development of the vortical structure long after the passage of the shock wave, and it is used throughout the computations. This spacing is also reasonable because it is consistent with the resolution of the experimental data of Haas (1984), Haas \& Sturtevant (1987) and Jacobs (1992), with which our results are compared.

\section{Numerical simulations of the flow}

Figure 4 shows density contour plots (at times $\bar{t}=0$ to 70 ) of the interaction of a $M=1.1$ shock, in air, with a single circular helium jet of light/heavy gas density ratio $\bar{\rho}_{L} / \bar{\rho}_{H}=0.138$. The flow corresponds closely with the schematic shown in figure 2 . The corresponding vorticity contour plots (figure 5) show the development of the vortex pair. In the first frame, at $\vec{t}=0$, the shock has not yet passed the jet, so no vorticity is present. Just after the shock has passed the jet (not shown in figure 5, but cf. figure $2 b$ ), the vorticity is concentrated primarily at the top and bottom of the jet. In the next frame, at $\bar{t}=10$, induced motions at the edges of the jet have swirled this vorticity into the innermost portions of the developing lobes. At $\bar{t}=20-50$, self-organization of the vorticity results in the emergence of distinct vortex cores.

The existence of vortex cores is also evident from cross-sectional slices through the structure. We expect low density, essentially uniform pressure, and high vorticity and mass fraction inside the core. We also expect the velocity distribution to be approximately that due to a solid-body rotation. Figure $6(a)$ shows actual data along a vertical cut slightly to the left of the vorticity centroid at $\bar{t}=90$. The cut is shown schematically in figure $6(b)$. The expectations are consistent with the data. In the case of $p$, note the fine scale: the maximum noise amplitude is less than $0.2 \%$. In the case of $\omega$, noise derives from its calculation as the simple central difference of velocity data.

For the flows considered here, vorticity is not the most useful variable for interpretation of the results. It is noisy and covers too broad a range of values, which are difficult to present in a contour plot. Density contours are a better choice, especially once it is understood that the flow is vortex-driven. They are smooth, cover a limited range of values, and are more closely associated with available experimental results (Haas 1984; Haas \& Sturtevant 1987; Jacobs 1992). They convey information about 


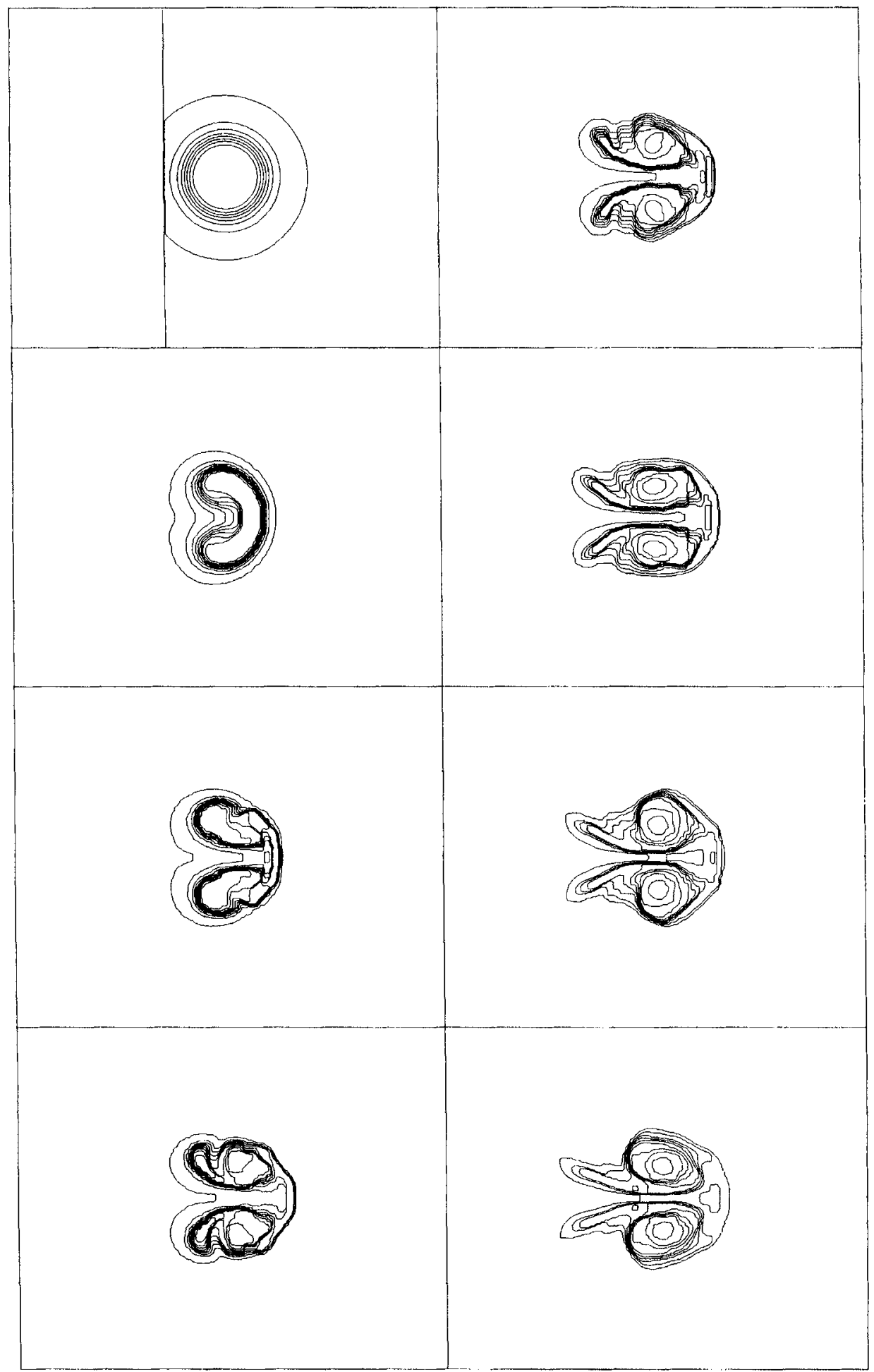

Figure 4. Density contour plots at $\bar{t}=0,10,20,30,40,50,60,70$ for $M=1.1$ and $\bar{\rho}_{r} / \bar{\rho}_{H}=0.138$. Each plot contains nine equally spaced contours spanning the $10 \%$ to $90 \%$ levels between $\bar{\rho}_{\text {min }}$ and $\bar{\rho}_{\text {max }}$ in the instantaneous flow field. The minimum and maximum levels are, respectively, $\left(\bar{\rho}_{\text {min }}, \bar{\rho}_{\text {max }}\right)=(0.138,1.169),(0.157,1.186),(0.158,1.178),(0.163,1.167),(0.173,1.165),(0.179,1.166)$, $(0.183,1.166)$, and $(0.187,1.166)$. 


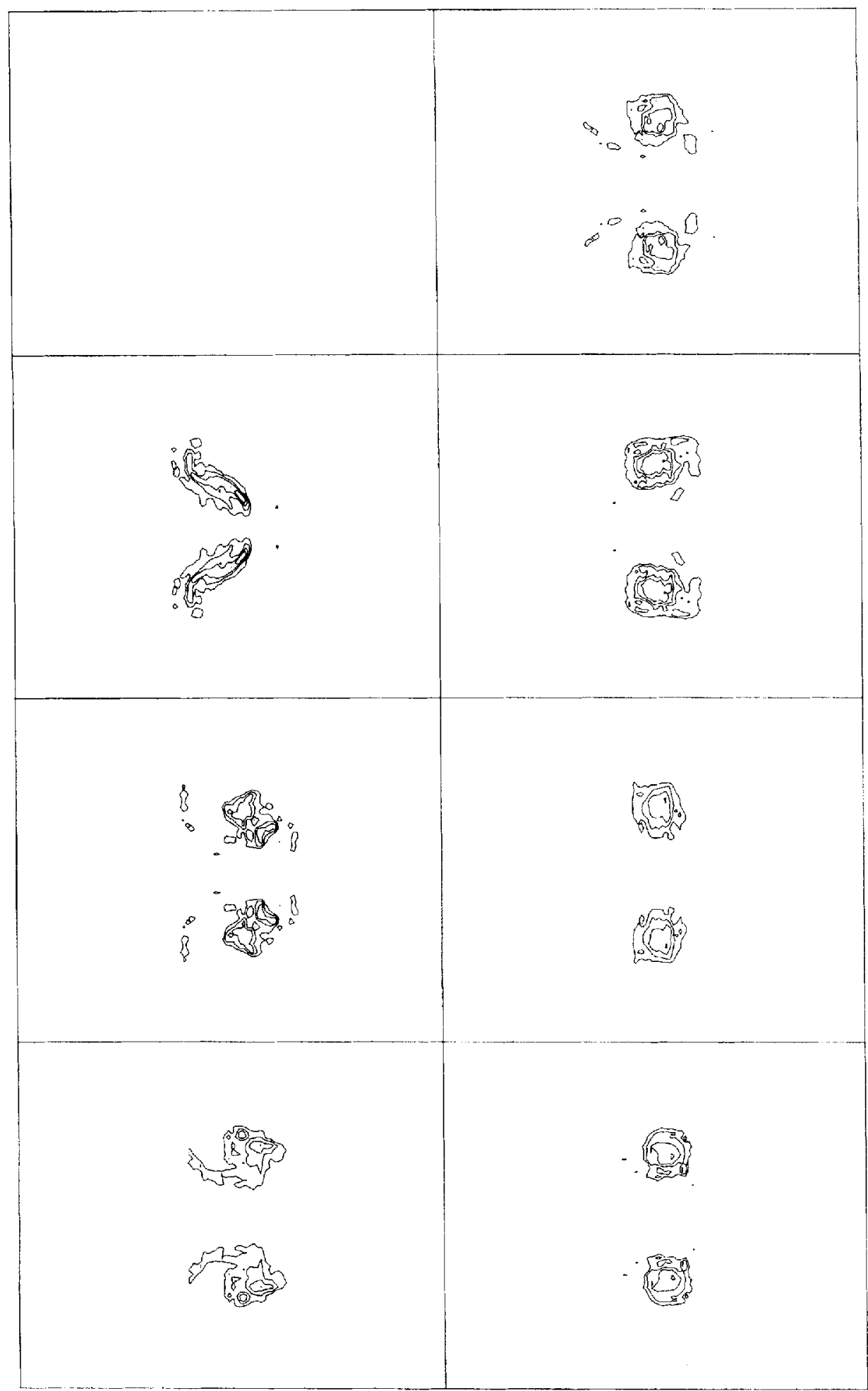

Figure 5. Vorticity contour plots at $\bar{t}=0,10,20,30,40,50,60,70$ for $M=1.1$ and $\bar{\rho}_{L} / \bar{\rho}_{H}=0.138$. Each plot contains four contours representing the $20 \%, 40 \%, 60 \%$, and $80 \%$ levels between 0 (negative vorticity contours are not shown) and $\bar{\omega}_{\max }$ in the instantaneous flow field. The maximum levels are, respectively, $\bar{\omega}_{\max }=0,1.499,1.712,2.003,1.768,1.494,1.652$, and 1.217 . The plots have been magnified $2 \times$ to show detail. 


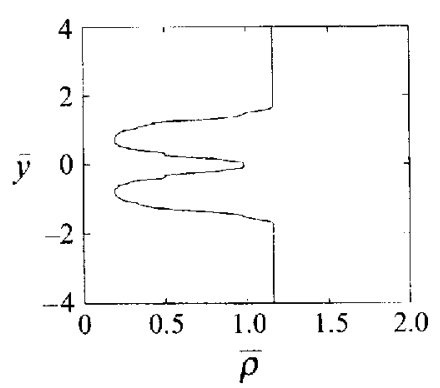

(a)
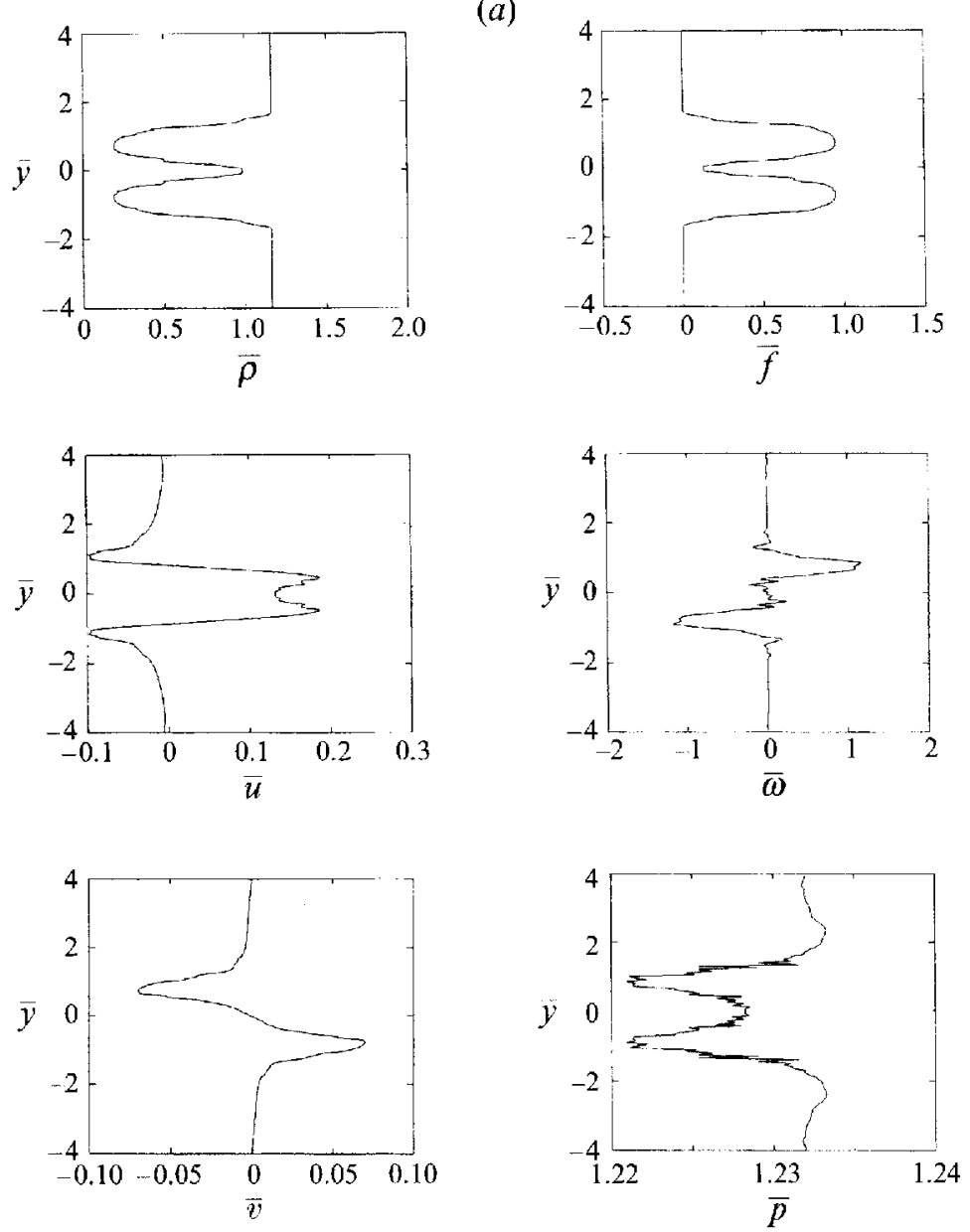

(b)

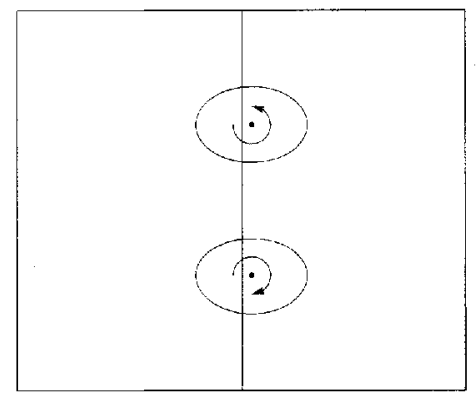

Figure 6. (a) Vertical cuts slightly to the left of the centre of vorticity of the $M=1.1$ and $\bar{\rho}_{L} / \bar{\rho}_{H}=0.138$ flow at $\bar{t}=90$. (b) Schematic of the vertical cuts shown in $(a)$.

organization and stabilization of the vorticity into a stable core, indirectly, through the circularization of contours. Finally, they provide information which the vorticity contours do not, especially about straining and motion of the light/heavy gas interface, which would be an important consideration for mixing applications. For these reasons, we primarily visualize the flows in terms of density, not vorticity, contour plots. 


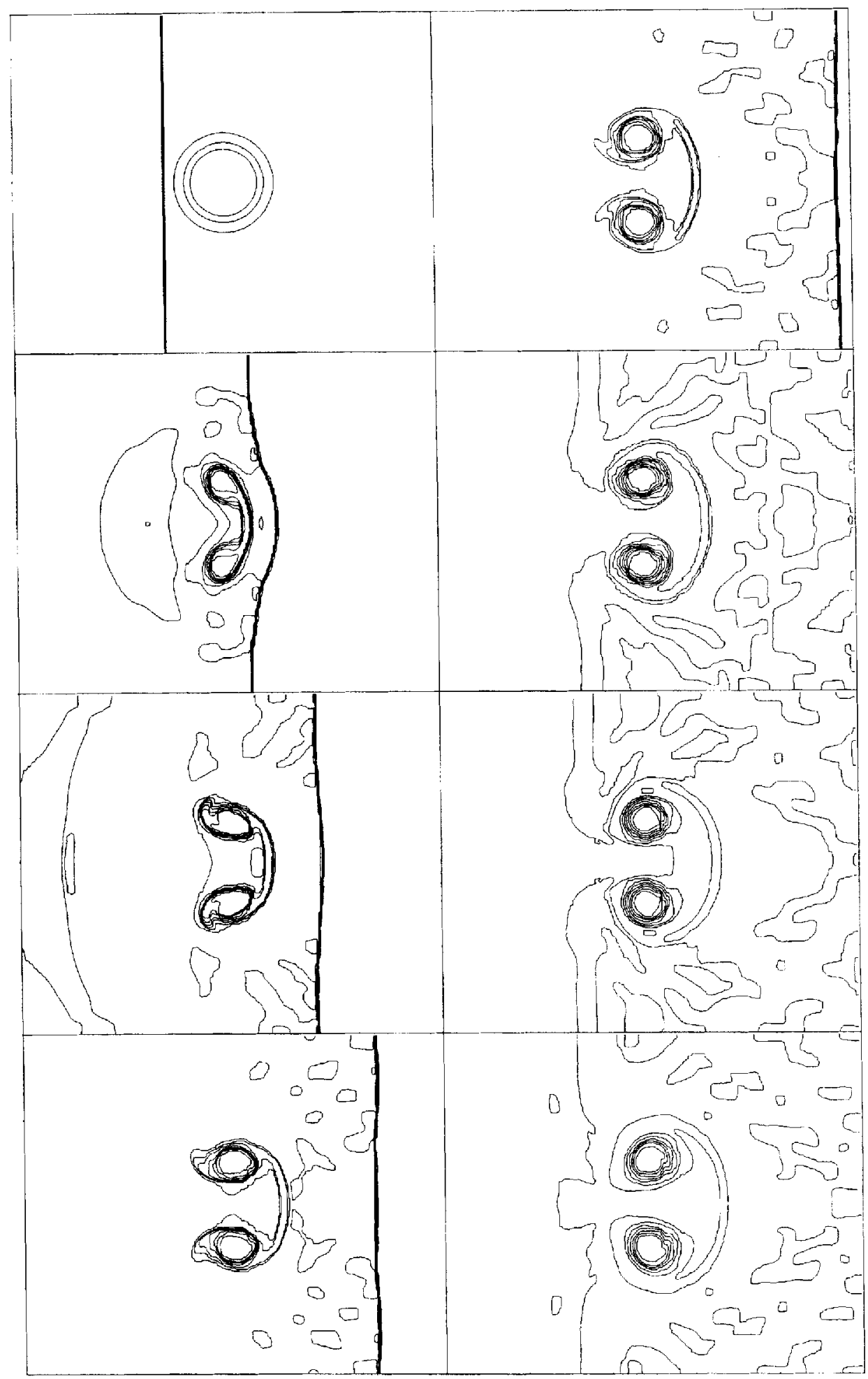

Figure 7 . Density contour plots at $\bar{t}=0,10,20,30,40,50,60,70$ for $M=2$ and $\bar{\rho}_{L} / \bar{\rho}_{H}=0.138$. Each plot contains nine equally spaced contours spanning the $10 \%$ to $90 \%$ levcls between $\bar{\rho}_{\min }$ and $\bar{\rho}_{\max }$ in the instantaneous flow field. The minimum and maximum levels are, respectively, $\left(\bar{\rho}_{\text {min }}, \bar{\rho}_{\text {max }}\right)=(0.138,2.667),(0.303,3.151),(0.349,3.092),(0.388,3.090),(0.394,3.146),(0.401,2.933)$, $(0.397,2.871)$, and $(0.356,2.671)$. 
For example, refer to figure 4 . At $\bar{t}=20-30$, that fluid which does not become part of the vortex core is pinched off as a pair of upstream tails. At the downstream end, a well-defined thin filament connects the two cores. This filament stretches and thins in the vertical direction due to a strong straining motion from the high-vorticity cores. Note the circularization of the vortex cores as the vortex stabilizes $(\bar{t}=40-50)$. The cores induce in each other a strong, horizontal induced velocity, thus moving downstream more rapidly than the tails, which stretch upstream as they trail behind $(\bar{t}=40-70)$. These tails also experience strong induced velocities which cause them to rotate towards the centreline.

Computations were performed for a number of shock strengths and light/heavy gas density ratios. In each case, the channel height and the initial jet interface thickness are fixed. The shock strength and light/heavy gas density ratio control the initial vorticity production, so changing these parameters should change the rate of development of the flow. However, the basic features are similar to the $M=1.1, \bar{\rho}_{L} / \bar{\rho}_{H}=0.138$ case discussed above. For example, figure 7 shows $\dagger$ the case $M=2.0$ and $\bar{\rho}_{L} / \bar{\rho}_{H}=0.138$ at times $\bar{t}=0-14$. The differences between this case and the $M=1.1$ case are twofold. First, the aspect ratio (height/width) of the structure is increased because of greater streamwise compression due to a stronger shock. Second, the increased shock strength increases the strength of the vortex pair. Overall, the resulting structure is more nearly an ideal vortex pair: the vortices are more compact and uniform in shape, and the tails are less prominent. In addition, development of the vortex occurs much more rapidly.

To quantitatively characterize a vortical flow, it is necessary to describe its strength, timescale, and motion. In all the canonical flow cases, development of the structure can be represented by the time history of the circulation about either vortex and the trajectory of the vortex pair. Consider again the case $M=1.1$ and $\bar{\rho}_{L} / \bar{\rho}_{H}=0.138$. The circulation about one of the vortical structures is shown in figure $8(a)$. It is calculated by evaluating the integral

$$
\bar{\Gamma}=\oint \bar{u} \cdot \mathrm{d} \bar{x}
$$

around a rectangular contour coincident with the upper half-plane of the flow field and moving with the developing vortex pair. The circulation rises rapidly as the shock passes through the jet, the peak (at $\bar{t}=4$ ) corresponding to the transmitted shock exiting the downstream end of the jet. At this time, the centre of the transmitted shock is curved outward where it has passed through the light-gas jet, while the top and bottom remain planar. Therefore, there is a net incremental positive contribution to $\bar{\Gamma}=\oint \bar{u} \cdot \mathrm{d} \bar{x}$ along the lower horizontal portion of the circulation contour from the fluid ahead of the jet but behind the shock. This represents a contribution due to shock curvature in addition to that caused by baroclinically generated vorticity.

Because the jet has not yet accelerated to its full vortex-induced velocity, it is moving slower than the fluid between the jet and the shock. As time goes on, this fluid is rapidly swept downstream out of the computational domain (and the circulation contour). Thus, the circulation should drop to only that associated with the baroclinically generated vorticity soon after the transmitted shock exits the domain.

In figure $8(a)$, the shock is so weak that the additional circulation due to shock curvature is negligible. However, it becomes significant in the case of stronger shocks or lower light/heavy gas density ratios. For example, consider $M=2.0$ and a

$\dagger$ All the plots contain nine equally spaced contours from near maximum to near minimum. The jet radius is always unity. At $\bar{t}=0$, variations in shock strength cause more or less of the intermediate contours to cluster at the shock or at the jet, changing their apparent sizes in the plot. 

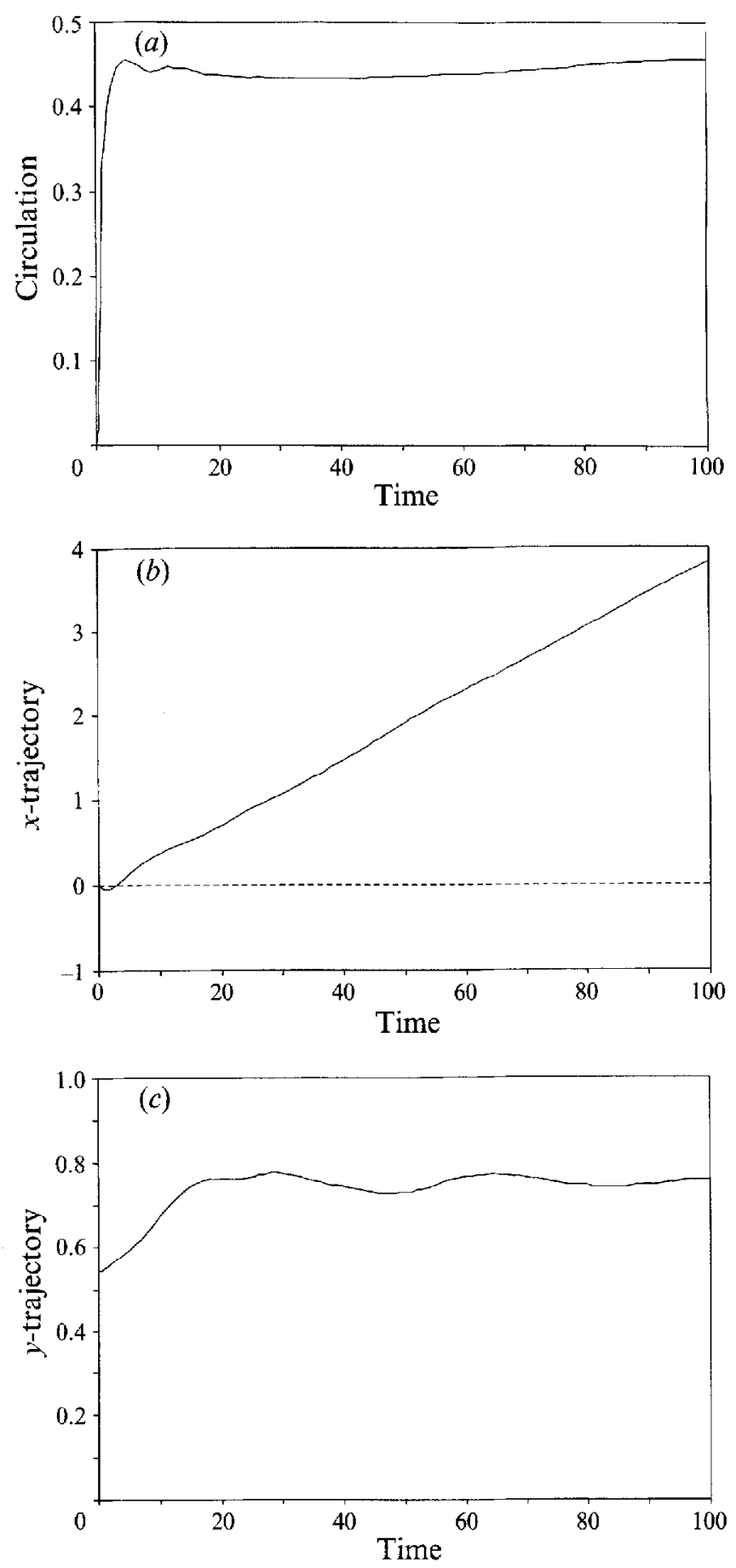

FIGURE 8. (a) Circulation, (b) $x$-trajectory, and (c) $y$-trajectory for the flow of figure 4.

light/heavy gas density ratio of 0.138 . In that case, the circulation reaches a peak instantaneous early-time value of 1.9 , then drops to a late-time value of 1.2 after the shock has left the domain. As expected, the difference of 0.7 exactly agrees with direct calculation of the additional $\bar{\Gamma}=\int \bar{u} \cdot \mathrm{d} \bar{x}$ due to the portion of the contour between the 


\begin{tabular}{ccccc}
\hline$M$ & $\bar{\rho}_{L} / \bar{\rho}_{H}$ & $\bar{\Gamma}$ & $\bar{y}_{\infty}$ & $\bar{U}$ \\
1.05 & 0.138 & 0.24 & 0.72 & 0.018 \\
1.1 & 0.138 & 0.45 & 0.75 & 0.033 \\
1.2 & 0.138 & 0.72 & 0.82 & 0.054 \\
1.5 & 0.138 & 0.97 & 1.00 & 0.063 \\
2.0 & 0.138 & 1.20 & 1.00 & 0.070 \\
1.1 & 0.354 & 0.272 & 0.82 & 0.0195 \\
1.1 & 0.569 & 0.162 & Not steady state & - \\
1.1 & 0.785 & 0.075 & Not steady state & - \\
2.0 & 0.354 & 0.765 & 1.20 & 0.0300 \\
2.0 & 0.569 & 0.448 & 1.20 & 0.0193 \\
2.0 & 0.785 & 0.205 & Not steady state & - \\
TABLE 1. Steady-state $\bar{\Gamma}, \bar{y}_{\infty}$, and $\bar{U}$ for the canonical flows \\
\hline \multicolumn{7}{c}{} \\
\hline
\end{tabular}

jet and the shock while the shock was in the domain. Thus the circulation due to baroclinically generated vorticity, which is the focus of this study, is automatically separated from the circulation associated with shock curvature after the shock exits the domain, and from that time onward, the circulation remains essentially constant.

Returning to the $M=1.1$ flow, the horizontal trajectory is shown in figure $8(b)$. We track the light gas, i.e. the location of the centre of mass fraction, relative to the motion of the ambient fluid behind the shock. This is defined as

$$
\bar{x}_{c m f} \equiv \frac{\int \bar{x} f \mathrm{~d} \bar{A}}{\int f \mathrm{~d} \bar{A}} .
$$

There is a small early-time dip just after the shock passes over the jet, when the still stationary jet appears to move backwards relative to the ambient fluid. This occurs because the jet initially behaves like a solid cylinder whose inertia prevents it from instantaneously acquiring the velocity behind the shock. However, the deposited vorticity soon accelerates the structure to a velocity faster than the ambient fluid. Minor oscillations occur until the structure stabilizes at around $\bar{t}=50$ to form vortex cores with trailing low-vorticity tails. Thereafter, the trajectory is very steady, the velocity of translation being essentially constant.

The vertical trajectory is likewise defined as the location of the centre of mass fraction, but taken over only the upper half-plane, i.e.

$$
\bar{y}_{c m f} \equiv \frac{\int \bar{y} f \mathrm{~d} \bar{A}}{\int f \mathrm{~d} \bar{A}} .
$$

Equivalently, this describes the half-spacing of the vortex pair. This is shown in figure $8(c)$. The trajectory shows a rapid initial increase, then levels off. After about $\bar{t}>20$, $\bar{y}_{c m f}$ is essentially constant, although there is a small, slowly decaying oscillation as the vortex continues to stabilize.

We seek to characterize the flow in an overall sense, rather than in fine detail. The essential constancy of circulation, $x$-velocity, and vortex half-spacing suggests that specification of these three parameters, along with a characteristic timescale, is 
sufficient to characterize the flow: $\bar{\Gamma} \approx 0.45, \bar{U} \equiv \mathrm{d} x_{c m f} / \mathrm{d} \bar{\tau} \approx 0.033$, and $\bar{y}_{\infty} \approx 0.75$. These values may similarly be determined for the other flows; they are summarized in table 1.

\section{Analytical models for the canonical problem}

\subsection{Strength of the vortex pair}

Since the circulation is due only to the initial interaction, it should be a function of only the shock strength and the density ratio. Various authors have attempted to model this relationship.

Rudinger \& Somers (1960) considered the interaction to consist of two steps. First, the jet is given an impulse by the passing shock which accelerates it to the velocity which a solid body of the same density would attain. There is no distortion of the jet during this step. Second, the jet is transformed into a vortex in a process modelled as the impulsive motion of an infinite lamina accelerated from rest. Rudinger \& Somers did not explicitly derive a formula for $\Gamma$, but their results can be manipulated to give

$$
\bar{\Gamma}=\frac{8}{\pi} \bar{y}_{\infty} \bar{v}_{2}\left(\frac{1-\bar{\rho}_{L} / \bar{\rho}_{H}}{1+\bar{\rho}_{L} / \bar{\rho}_{H}}\right),
$$

where $\bar{v}_{2}$ is the velocity of the air behind the shock.

Picone et al. (1985) and Picone \& Boris (1988) analytically integrated the vorticity equation for a shock passing through a circular cross-section, and argued that

$$
\bar{\Gamma}=2 \bar{v}_{2}\left(\frac{\bar{v}_{2}}{2 M}-1\right) \ln \left(\frac{\bar{\rho}_{L}}{\bar{\rho}_{H}}\right) .
$$

However, their computations showed that the model overpredicted the circulation by a factor of about two.

Most recently, using simple one-dimensional gasdynamics, Hendricks \& Marble (1991) derived coupled, nonlinear equations that could be solved numerically for the circulation as a function of Mach number and density ratio. They also presented computations of two low-Mach-number cases showing good agreement with their model. The disadvantages of this method are that the dependence on the shock strength and density ratio cannot be easily visualized and that the equations are tedious, although straightforward, to solve numerically.

In summary, the currently available scaling laws either do not accurately predict the circulation or are difficult to use and interpret. We seek a circulation model which overcomes these deficiencies. The interaction of a shock and a curved jet interface is difficult to model exactly. For example, during shock passage, the intersection of the shock with the perimeter separates an undisturbed circular boundary ahead of the shock from a non-circular boundary behind the shock (figure 9). Also, the shock wave is planar outside the jet, but is curved inside the jet, due to the light gas there. Hawley \& Zabusky (1989) have demonstrated the use of shock polar analysis to obtain detailed and accurate information about shock-interface interactions. That technique avoids the above difficulties, but does not meet the present goal of a simple, easy-to-use, analytical expression. At the expense of mathematical rigour, we seek an approximate relation as follows.

Consider a cross-section through an initially circular jet, as shown in figure 10 . The light/heavy gas density ratio is $\rho_{L} / \rho_{H}$ and the density and pressure ratios across the shock wave are $\rho_{2} / \rho_{1}$ and $p_{2} / p_{1}$. Following Rudinger \& Somers (1960) and Picone \& 


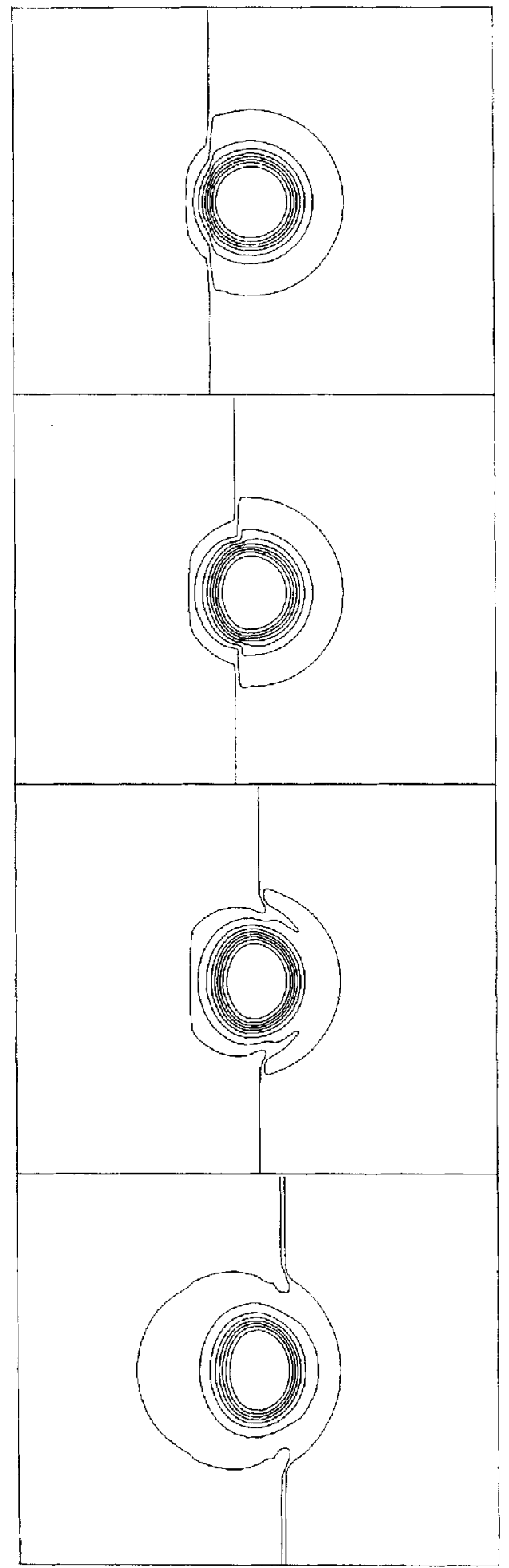

Figure 9. Density contour plots at $\bar{t}=0.5,1.0,1.5,2.0$ for $M=1.1$ and $\bar{\rho}_{L} / \bar{\rho}_{H}=0.138$. Each plot contains nine equally spaced contours spanning the $10 \%$ to $90 \%$ levels between $\bar{\rho}_{m i n}$ and $\bar{\rho}_{\text {max }}$ in the instantaneous flow field. The minimum and maximum levels are, respectively, $\left(\bar{\rho}_{\text {min }}, \vec{\rho}_{\text {max }}\right)=(0.138,1.171),(0.146,1.171),(0.152,1.190)$, and $(0.154,1.253)$. 


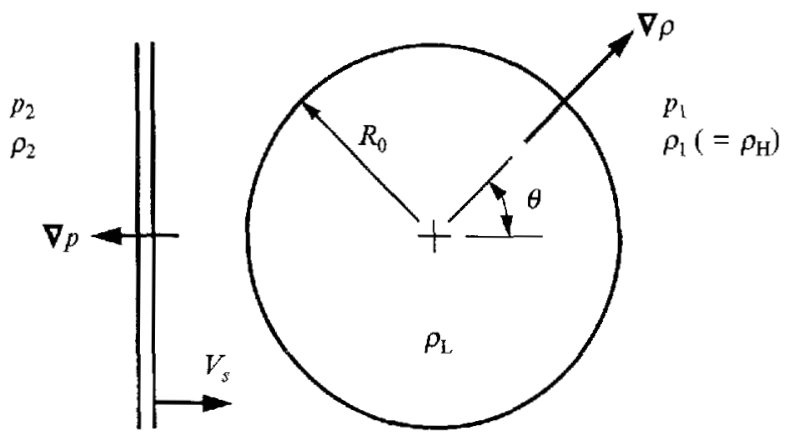

FIGURE 10. Schematic for circulation and impulse models.

Boris (1988), we approximate the jet boundary as circular during passage of the shock. The interface is idealized as a sharp step discontinuity in density. The shock is idealized as a planar discontinuity moving with constant velocity.

The vorticity production term of the vorticity equation is

$$
\frac{\mathrm{D} \omega}{\mathrm{D} t}=\frac{1}{\rho^{2}}(\nabla \rho \times \nabla p)+\ldots
$$

Integration over the time of shock passage gives the vorticity, $\omega=\omega \hat{\boldsymbol{e}}_{z}$, which is perpendicular to the plane of the jet:

$$
\omega=\int_{0}^{\infty} \frac{1}{\rho^{2}}|\nabla \rho \times \nabla p| \mathrm{d} t .
$$

The density terms in the integrand are difficult to model properly within the context of a simple analytical model. Instead, let us assume that the effect of density can be approximated by suitably chosen values taken outside the integral. Thus,

$$
\begin{aligned}
\omega & \approx \frac{1}{\rho^{2}} \int_{0}^{\infty}|\nabla \rho||\nabla p| \sin \theta \mathrm{d} t \\
& \approx \frac{1}{\rho^{2}} \int_{0}^{\infty} \Delta \rho \delta\left(r-R_{0}\right) \Delta p \delta\left(x-V_{s} t\right) \sin \theta \mathrm{d} t \\
& \approx \frac{1}{V_{s}} \frac{\Delta \rho}{\rho} \frac{\Delta p}{\rho} \delta\left(r-R_{0}\right) \sin \theta .
\end{aligned}
$$

Integrating over the area gives the circulation:

$$
\begin{aligned}
\Gamma & =\iint \omega \mathrm{d} A \\
& \approx \frac{1}{V_{s}} \frac{\Delta \rho}{\rho} \frac{\Delta p}{\rho} \int_{r=\emptyset}^{\infty} \int_{\theta=0}^{\pi} \delta\left(r-R_{0}\right) \sin \theta r \mathrm{~d} r \mathrm{~d} \theta \\
& \approx \frac{2 R_{0}}{V_{s}} \frac{\Delta p}{\rho} \frac{\Delta \rho}{\rho} .
\end{aligned}
$$

The denominator contains two adjustable values of $\rho$, which must be chosen in such a way as to correlate the data. Since the pressure gradient is due to the shock, we 


\begin{tabular}{|c|c|c|c|c|c|c|}
\hline \multirow[b]{2}{*}{$M$} & \multicolumn{2}{|c|}{ Computed } & \multicolumn{4}{|c|}{ Predicted } \\
\hline & $\bar{\rho}_{L} / \bar{\rho}_{H}$ & $\bar{\Gamma}$ & $\bar{\Gamma}(\mathrm{R}-\mathrm{S})$ & $\bar{\Gamma}(\mathrm{P}-\mathrm{B})$ & $\bar{\Gamma}(\mathrm{H}-\mathrm{M})$ & $\Gamma(\mathrm{Y}-\mathrm{K}-\mathrm{Z})$ \\
\hline 1.05 & 0.138 & 0.240 & 0.113 & 0.310 & 0.196 & 0.228 \\
\hline 1.1 & 0.138 & 0.450 & 0.230 & 0.584 & 0.376 & 0.412 \\
\hline 1.2 & 0.138 & 0.720 & 0.483 & 1.056 & 0.699 & 0.690 \\
\hline 1.5 & 0.138 & 0.970 & 1.339 & 2.113 & 1.455 & 1.130 \\
\hline 2.0 & 0.138 & 1.200 & 2.410 & 3.402 & 2.338 & 1.420 \\
\hline 1.1 & 0.354 & 0.272 & 0.159 & 0.318 & 0.239 & 0.260 \\
\hline 1.1 & 0.569 & 0.162 & 0.098 & 0.172 & 0.144 & 0.150 \\
\hline 1.1 & 0.785 & 0.075 & 0.038 & 0.074 & 0.069 & 0.066 \\
\hline 2.0 & 0.354 & 0.765 & 1.824 & 1.787 & 1.415 & 0.896 \\
\hline 2.0 & 0.569 & 0.448 & 1.049 & 0.969 & 0.863 & 0.515 \\
\hline 2.0 & 0.785 & 0.205 & 0.461 & 0.417 & 0.453 & 0.226 \\
\hline
\end{tabular}

TABLE 2. Computed and predicted $\bar{\Gamma}$ for the canonical flows: R-S, Rudinger \& Somers (1960); P-B, Picone \& Boris (1988); H-M, Hendricks \& Marble (1991); Y-K-Z, present model

choose $\rho_{2}$ for the pressure term, and since the density gradient is across the interface, we choose the average density at the interface, $\frac{1}{2}\left(\rho_{L}+\rho_{H}\right)$, for the density term. The circulation then becomes

Non-dimensionalizing gives

$$
\Gamma \approx \frac{4 R_{0}}{V_{s}} \frac{\Delta p}{\rho_{2}}\left(\frac{\Delta \rho}{\rho_{L}+\rho_{I}}\right) .
$$

$$
\bar{\Gamma} \approx \frac{4}{\gamma_{1} M}\left(\frac{\bar{\rho}_{1}}{\bar{\rho}_{2}}\right)\left(\frac{\bar{p}_{2}}{\bar{\rho}_{1}}-1\right)\left(\frac{1-\bar{\rho}_{L} / \bar{\rho}_{H}}{1+\bar{\rho}_{L} / \bar{\rho}_{H}}\right),
$$

where

$$
\frac{\bar{\rho}_{2}}{\bar{\rho}_{1}}=\frac{\left(\gamma_{1}+1\right) M^{2}}{\left(\gamma_{1}-1\right) M^{2}+2}
$$

and

$$
\frac{\bar{p}_{2}}{\bar{p}_{1}}=1+\left(\frac{2 \gamma_{1}}{\gamma_{1}+1}\right)\left(M^{2}-1\right) \text {. }
$$

This expression idealizes the shock wave and jet interfaces as simple step-function distributions, but in a real flow, these will have finite thicknesses. To examine these effects, the model was modified to represent the shock and jet interface thicknesses using a hyperbolic tangent function with an adjustable constant. This allowed consideration of a family of interfaces having the same difference in density or pressure as in the step-function case, but spread over a finite spatial distance. In the case of the shock thickness, the result was always identical to the step-function distribution, regardless of the shock thickness used. In the case of jet interface thickness, the change in circulation was negligible. For example, for a $10 \%-90 \%$ interface thickness of 0.9 ( $90 \%$ of the jet radius), the difference from the step-function case was only $0.3 \%$. This suggests that for fixed differences in density and pressure, even as the gradients vary due to changing interface thickness, integration gives a circulation which is essentially independent of the gradient magnitudes.

A comparison of predicted and computed data is shown in table 2 for the cases which were computed to steady state. The Hendricks \& Marble, Picone \& Boris, and Rudinger \& Somers models exhibit varying degrees of success at low Mach numbers, but overpredict the circulation by as much as a factor of two at high Mach numbers. 
(a)

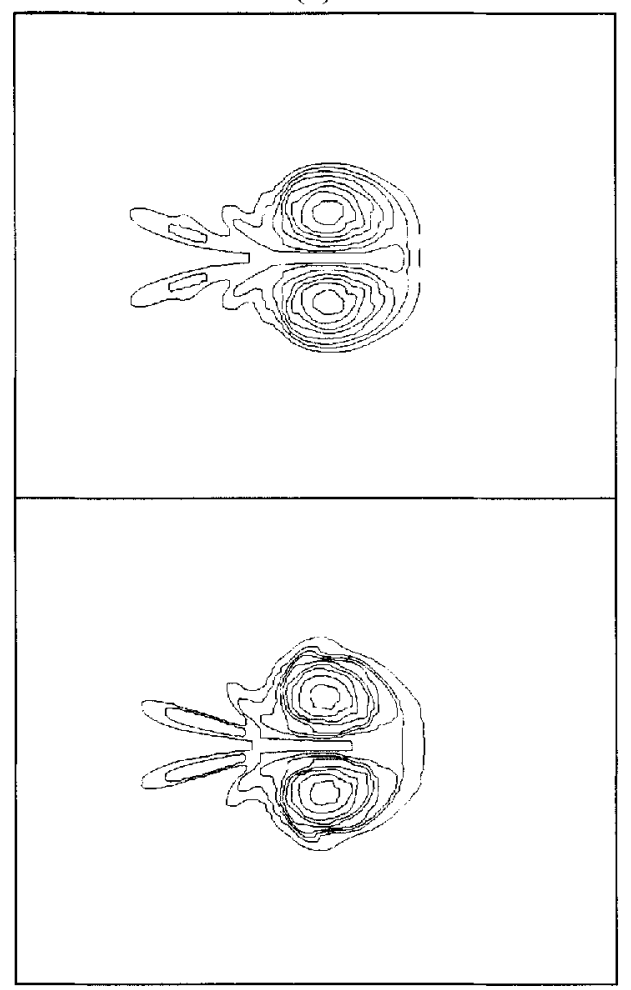

(b)

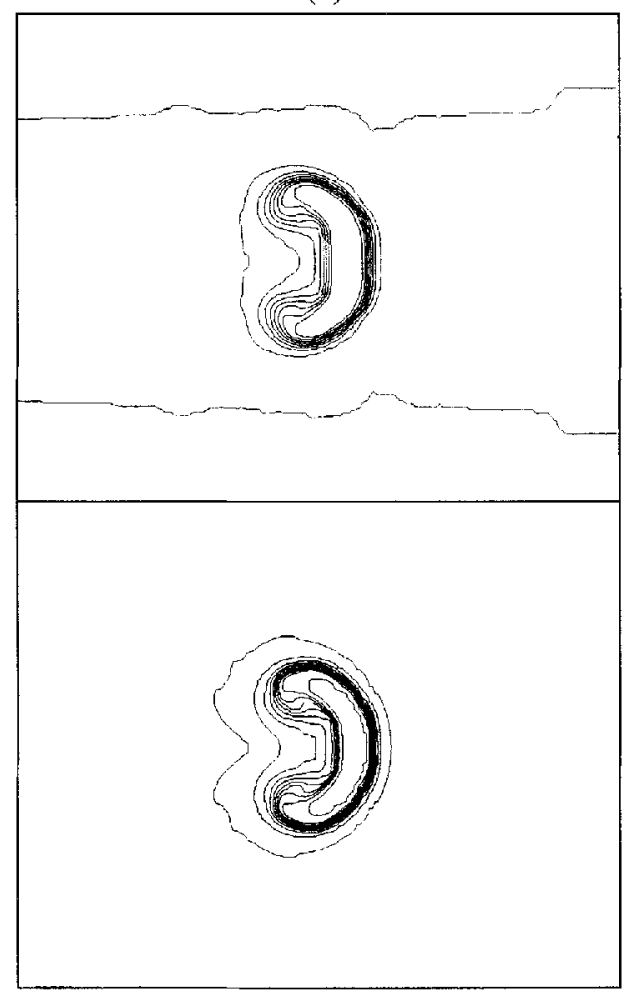

FIGURE 11. (a) Density contours for $\bar{\rho}_{L} / \bar{\rho}_{H}=0.138$; (top) $M=1.05$ at $\bar{t}=200$ and (bottom) $M=1.1$ at $\bar{t}=100$. Each plot contains nine equally spaced contours spanning the $10 \%$ to $90 \%$ levels between $\bar{\rho}_{\text {min }}$ and $\bar{\rho}_{\text {manx }}$ in the instantaneous flow field. The minimum and maximum levels are, respectively, $\left(\bar{\rho}_{\min }, \bar{\rho}_{\max }\right)=(0.199,1.084)$ and $(0.193,1.164) .(b)$ Density contours for $M=1.1:($ top $) \bar{\rho}_{L} / \bar{\rho}_{H}=0.785$ at $\bar{t}=90$ and (bottom) $\bar{\rho}_{L} / \bar{\rho}_{i z}=0.569$ at $\bar{t}=40$. Each plot contains nine equally spaced contours spanning the $10 \%$ to $90 \%$ levels between $\bar{\rho}_{\min }$ and $\bar{\rho}_{\text {max }}$ in the instantaneous flow field. The minimum and maximum levels are, respectively, $\left(\bar{\rho}_{\text {min }}, \bar{\rho}_{\text {max }}\right)=(0.894,1.170),(0.649,1.171)$.

The present model correlates the predicted and computed values to within about $15 \%$. More importantly, it requires only three easily obtained parameters (the initial jet radius, shock strength, and light/heavy gas density ratio), and is easy to interpret and use. Although mathematically approximate, it retains the essential features of the shock-interface interaction, through what may be termed 'dimensional analysis with correlation'.

\subsection{Characteristic time for development}

Visual comparison of contour plots allows determination of the relative timescales at which different flows evolve. For example, figure $11(a)$ shows that for $\bar{\rho}_{L} / \bar{\rho}_{H}=0.138$, $M=1.05$ at $\bar{t}=200$ and $M=1.1$ at $\bar{t}=100$ are at qualitatively similar stages of development. Similarly, figure $11(b)$ shows that for $M=1.1, \bar{\rho}_{L} / \bar{\rho}_{H}=0.785$ at $\bar{t}=90$ and $\bar{\rho}_{L} / \bar{\rho}_{H}=0.569$ at $\bar{t}=40$ are qualitatively similar.

Quantitatively, a characteristic time for the interaction can be formulated as

$$
\tau=\frac{H W}{\Gamma}
$$

where $H$ is a characteristic height, $W$ is a characteristic width, and $\Gamma$ is the circulation. For $H$ we take $R_{0}$, the vertical dimension, and for $W$ we take $\left(\rho_{1} / \rho_{2}\right) R_{0}$, the horizontal 
dimension just after the shock has passed (the factor $\rho_{1} / \rho_{2}$ accounts for the streamwise compression due to the shock). Therefore,

$$
\tau=\frac{\rho_{1}}{\rho_{2}} \frac{R_{0}^{2}}{\Gamma}
$$

Substituting the previous expressions for $\bar{\Gamma}$ and $\rho_{2} / \rho_{1}$ and non-dimensionalizing gives

$$
\bar{\tau} \approx\left(\frac{\gamma_{1}+1}{8}\right)\left(\frac{M}{M^{2}-1}\right)\left(\frac{1+\bar{\rho}_{L} / \bar{\rho}_{H}}{1-\bar{\rho}_{L} / \bar{\rho}_{H}}\right)
$$

We note from visual interpretation of the contour plots that the time required for any of the flows to become fully developed is approximately $25 \bar{\tau}$. Further, the ratio of any two characteristic times verifies the relative timescales determined from qualitative comparison of the contour plots. For example, corresponding to figure $11(a)$, $\bar{\tau}(M=1.05) / \bar{\tau}(M=1.1)=1.96$, so that $M=1.05$ at $\bar{t}=196$ and $M=1.1$ at $\bar{t}=100$ represent similar stages of development. Similarly, corresponding to figure $11(b)$, $\bar{\tau}\left(\bar{\rho}_{L} / \bar{\rho}_{H}=0.785\right) / \bar{\tau}\left(\bar{\rho}_{L} / \bar{\rho}_{H}=0.569\right)=2.3$, so that $\bar{\rho}_{L} / \bar{\rho}_{H}=0.785$ at $\bar{t}=92$ and $\bar{\rho}_{L} / \bar{\rho}_{H}=0.569$ at $\bar{t}=40$ represent similar stages of development.

\subsection{Motion of the vortex pair}

The late-time structure resulting from the interaction of a shock wave and a single jet is essentially a vortex pair with finite core size, in a channel of finite height. Relative to the ambient fluid, the structure moves downstream with a velocity due to the induced motion of each vortex by the other.

The simplest model for the motion of a vortex pair, potential flow of a point vortex pair in an unbounded domain, predicts the normalized velocity to be $\bar{U} \bar{y}_{\infty} / \bar{\Gamma}=1 /(4 \pi)$. This would be the velocity in the simultaneous limits of core size tending toward zero and channel spacing tending toward infinity; it represents an upper limit for the velocity in an actual flow. As either the core size or channel spacing becomes finite, the velocity must decrease below $\bar{U}_{\bar{y}_{\infty} /} / \bar{\Gamma}=1 /(4 \pi)$. We seek a closed-form analytical model which describes the effects of both these phenomena on the motion of the vortex pair. These effects are first treated separately and then in combination.

\subsubsection{Effect of finite channel spacing}

Consider the effect of channel spacing alone. This problem consists of a pair of point vortices in a bounded domain. We treat the walls mathematically as lines of reflection. As shown in figure 12, the flow is equivalent to an infinite array of vortex pairs of strength $\pm \bar{\Gamma}$, each of spacing $2 \bar{y}_{\infty \alpha}$, in a channel of height $2 \bar{h}$. The actual vortex pair divides the domain into 'upper' and 'lower' halves, and the image vortex pairs are referred to as 'first upper', 'second upper', $\ldots$ and 'first lower', 'second lower', $\ldots$ respectively.

The upper actual vortex experiences induced-velocity contributions from each of the other vortices, as follows. The lower actual vortex induces a velocity

$$
\frac{\bar{\Gamma}}{2 \pi} \frac{1}{2 \bar{y}_{\infty}}
$$

the $n$th upper image pair induces a velocity

$$
\frac{\bar{\Gamma}}{2 \pi}\left[\frac{1}{2 n \bar{h}}-\frac{1}{2 n \bar{h}-2 \bar{y}_{\infty}}\right]
$$




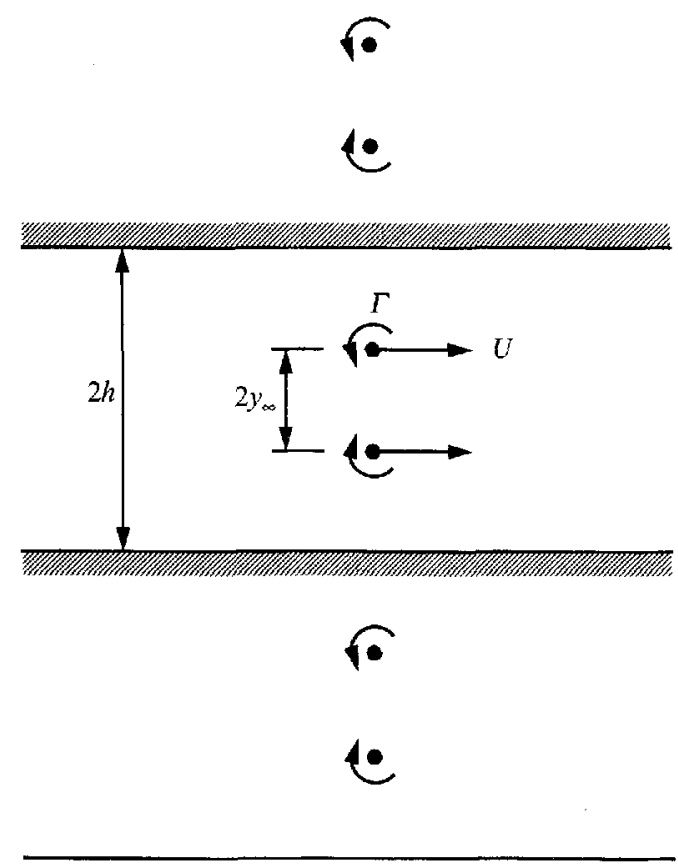

FIGURE 12. Schematic of point-vortex pairs in a channel.

and the $n$th lower image pair induces a velocity

The total induced velocity is

$$
\frac{\bar{\Gamma}}{2 \pi}\left[\frac{1}{2 n \bar{h}+2 \bar{y}_{\infty}}-\frac{1}{2 n \bar{h}}\right] .
$$

$$
\bar{U}=\frac{\bar{\Gamma}}{2 \pi}\left[\frac{1}{2 \bar{y}_{\infty}}+\sum_{n=1}^{\infty}\left\{\frac{1}{2 n \bar{h}}-\frac{1}{2 n \bar{h}-2 \bar{y}_{\infty}}\right\}+\sum_{n=1}^{\infty}\left\{\frac{1}{2 n \bar{h}+2 \bar{y}_{\infty}}-\frac{1}{2 n \bar{h}}\right\}\right]
$$

which may be simplified to $\quad \bar{U}=\frac{\bar{\Gamma}}{4 h} \operatorname{cotan}\left(\pi \frac{\bar{y}_{\infty}}{\bar{h}}\right)$,

or

$$
\frac{\bar{U} \bar{y}_{\infty}}{\bar{\Gamma}}=\frac{1}{4} \frac{\bar{y}_{\infty}}{\bar{h}} \operatorname{cotan}\left(\pi \frac{\bar{y}_{\infty}}{\bar{h}}\right) \text {. }
$$

As $\bar{y}_{\infty} / \bar{h}$ increases, i.e. as the channel spacing decreases, the velocity decreases. As $\bar{y}_{\infty} / \bar{h}$ tends towards zero, the unbounded point vortex result, $\bar{U} \bar{y}_{c c} / \bar{\Gamma}=1 /(4 \pi)$, is recovered.

\subsubsection{Effect of finite core size}

Consider the effect of finite core size alone. The model for this flow is a vortex pair with constant vorticity inside a finite core, in an unbounded domain. The vortex pair moves steadily, without change of shape or velocity, through an ambient fluid at rest at infinity. The simplifying assumption of constant vorticity, although not realizable in an actual flow, should be a reasonable approximation when the vortex core is large compared to the vorticity gradient at the edge of the region.

This problem has been investigated numerically by a number of authors. Pierrehumbert (1980) was the first to exhibit a family of solutions characterized by a 
single parameter $\bar{R} / \bar{y}_{\infty}$, the ratio of effective core size, $\bar{R}=(\bar{A} / \pi)^{\frac{1}{2}}$, to centroid-tocentreline spacing, $\bar{y}_{\infty}$. The problem has also been solved analytically using a perturbation analysis with parameter $\epsilon \equiv \bar{R} / \tilde{y}_{\infty}$. Yang \& Kubota (1993) reported the normalized velocity to be

$$
\frac{\bar{U} \bar{y}_{\infty}}{\bar{\Gamma}}=\frac{1}{4 \pi}\left[1-\frac{1}{8}\left(\frac{\bar{R}}{\bar{y}_{\infty}}\right)^{4}+O\left(\left(\frac{\bar{R}}{\bar{y}_{\infty}}\right)^{6}\right)\right] .
$$

The analytical solution has two advantages over the numerical solution. First, it may be evaluated directly for a specified value of $\bar{R} / \bar{y}_{\kappa}$. Second, it illustrates the physics of the solution, in particular the order in $\bar{R} / \bar{y}_{\infty}$ at which the velocity first begins to deviate from the classical limit.

The above result assumes an incompressible flow. Moore \& Pullin (1987) demonstrated numerically and analytically that the effect of compressibility is to reduce the velocity of a finite-core-size vortex pair in an unbounded domain. The highest-compressibility case of the present investigation, $M=2$ and $\bar{\rho}_{L} / \bar{\rho}_{H}=0.138$, is bounded by Moore \& Pullin's 'evacuated vortex', which for corresponding conditions, has a normalized velocity only $1.6 \%$ below the classical $1 /(4 \pi)$ value. Therefore, the effects of compressibility in the present study are negligible.

\subsubsection{Combined effects of channel spacing and core size}

The effect of channel spacing alone may be represented as

$$
\frac{\bar{U} \bar{y}_{\infty}}{\bar{\Gamma}}=\frac{1}{4 \pi} f\left(\frac{\bar{y}_{\infty}}{\bar{h}}\right),
$$

where $f\left(\bar{y}_{\infty} / \bar{h}\right)$ represents the dependence on channel spacing. The effect of core size alone may be represented as

$$
\frac{\bar{U} \bar{y}_{\infty}}{\bar{\Gamma}}=\frac{1}{4 \pi} g\left(\frac{\bar{R}}{\bar{y}_{\infty}}\right),
$$

where $g\left(\bar{R} / \bar{y}_{\infty}\right)$ represents the dependence on core size.

A composite solution must simultaneously incorporate effects of channel spacing and core size: image vortices representing the channel-spacing effects must be accounted for in the perturbation analysis for core size, resulting in a two-parameter perturbation expansion. This is mathematically intractable. At the expense of mathematical rigour, we assume that the dominant contribution of the image vortices is through their point-vortex representations and obtain an approximate solution by simply substituting either of the solutions for the unperturbed-velocity term of the other. Thus,

or

$$
\begin{gathered}
\frac{\bar{U} \bar{y}_{\infty}}{\bar{\Gamma}}=\frac{1}{4 \pi} f\left(\frac{\bar{y}_{\infty}}{\bar{h}}\right) g\left(\frac{\bar{R}}{\bar{y}_{\infty}}\right) \\
\frac{\bar{U} \bar{y}_{\infty}}{\bar{\Gamma}} \approx \frac{1}{4 \pi} \frac{\pi \bar{y}_{\infty}}{\bar{h}}\left[\operatorname{cotan}\left(\frac{\pi \bar{y}_{\infty}}{\bar{h}}\right)\right]\left[1-\frac{1}{8}\left(\frac{\bar{R}}{\bar{y}_{\infty}}\right)^{4}\right] .
\end{gathered}
$$

This represents a simple, approximate model for the effects of both channel spacing and core size. It can be tested using data from the computations of table 1. Notice that the core size and spacing are quantities that are not determined a priori, but rather are determined from the contour plots. (However, the predicted values of $\bar{U}_{\bar{y}_{\infty}} / \bar{\Gamma}$ use a 


\begin{tabular}{lccccc} 
& & & \multicolumn{2}{c}{$\bar{U}_{\bar{y}_{\infty}} / \bar{\Gamma}$} \\
\cline { 5 - 6 }$M$ & $\bar{\rho}_{L} / \bar{\rho}_{H}$ & $\bar{R} / \bar{y}_{\infty}$ & $\bar{y}_{\infty} / \bar{h}$ & Computed & Predicted \\
1.05 & 0.138 & 1.0540 & 0.1800 & 0.0540 & 0.0600 \\
1.1 & 0.138 & 0.9866 & 0.1875 & 0.0550 & 0.0618 \\
1.2 & 0.138 & 0.8808 & 0.2000 & 0.0600 & 0.0636 \\
1.5 & 0.138 & 0.6693 & 0.2375 & 0.0617 & 0.0626 \\
2.0 & 0.138 & 0.5470 & 0.2500 & 0.0583 & 0.0618 \\
1.1 & 0.354 & 0.8982 & 0.2050 & 0.0588 & 0.0627 \\
2.0 & 0.354 & 0.4558 & 0.3000 & 0.0471 & 0.0542 \\
2.0 & 0.569 & 0.4443 & 0.3000 & 0.0517 & 0.0542
\end{tabular}

TABLE 3. Computed and predicted $\bar{U}_{y_{\circ} /} / \bar{\Gamma}$ for the canonical flows

\begin{tabular}{ccccccc} 
Channel & & & & & \multicolumn{2}{c}{$\bar{U}_{\bar{y}_{\infty}} / \bar{\Gamma}$} \\
spacing & $\bar{\Gamma}$ & $\bar{U}$ & $\bar{y}_{\infty}$ & $\bar{R}$ & Computed & Predicted \\
16 & 0.46 & 0.041 & 0.725 & 0.736 & 0.065 & 0.067 \\
8 & 0.45 & 0.033 & 0.753 & 0.740 & 0.055 & 0.062 \\
4 & 0.39 & 0.016 & 0.727 & 0.734 & 0.031 & 0.036
\end{tabular}

TABLE 4. Computed and predicted $\bar{U} \bar{y}_{\infty} / \bar{\Gamma}$ for variations in channel spacing

computed value for $\bar{R} / \bar{y}_{\infty}$ due to an inability to accurately model $\bar{y}_{\infty}$, cf. $\S 5.4$ below.) The effective vortex core radius is computed as

where

$$
\begin{aligned}
& \bar{R} \equiv(A / \pi)^{\frac{1}{2}}, \\
& \bar{A} \equiv \int f \mathrm{~d} \bar{A}
\end{aligned}
$$

is the mass-fraction-weighted area. The results are listed in table 3 . The predictions are somewhat higher than the computations, but the qualitative trends are well represented. For example, at fixed $\bar{\rho}_{L} / \bar{\rho}_{H}$, both show a slightly downward concave dependence on $M$.

\subsubsection{Additional tests of the velocity model}

In table 3 , the velocity model was only tested against a relatively small range of vortex spacing/channel spacing (less than $30 \%$ variation). Explicit variation in channel spacing provides a more rigorous and direct test. Three cases are computed: channel spacing $=16,8$, and 4 (the spacing $=8$ case is just the canonical flow of figure 4). The Mach number is $M=1.1$, and the density ratio is $\bar{\rho}_{L} / \bar{\rho}_{H}=0.138$. Qualitatively, these flows are all very similar, so additional contour plots are not shown. Quantitatively, the flows are again characterized by circulation, velocity, and spacing. Table 4 compares the steady-state computations and predictions. There is good agreement between computed and predicted values of normalized velocity. Note that the computed circulation values are essentially the same for the two wide-channel spacings but drop about $13 \%$ for the narrow-channel spacing. The circulation model, being independent of channel spacing, does not capture this trend, but it is still satisfactory as these are relatively small differences relative to a fourfold variation in channel spacing. 


\subsection{Spacing of the vortex pair}

In previous sections, the vortex half-spacing used in the velocity model was determined from the computations. We would like to have an analytical model for this quantity as well. Representation of the computed flow by the model flow requires the correspondence of both circulation and spacing. Dimensionally, the product of these quantities suggests a measure of the steady-state impulse of the flow:

$$
\bar{I}_{x}^{\prime}=\bar{\rho}_{m o d e l} \bar{y}_{\infty} \bar{\Gamma}
$$

where $\bar{\rho}_{\text {model }}$ is an appropriate density for the model flow. This is not exactly a true impulse, which is properly defined as an integral, but is a closely related quantity. The unspecified density gives the degree of freedom to match $\bar{I}_{x}^{\prime}$ for the constant-vorticity model to the initial impulse $\bar{I}_{x}\left(\bar{t}=0^{+}\right)$caused by the passage of a shock over a circular jet:

$$
\bar{I}_{x}\left(\bar{t}=0^{+}\right) \approx \bar{I}_{x}^{\prime}
$$

Without this freedom there would be no way to correlate the two quantities. The reason is that a uniform-vorticity vortex pair cannot have exactly the same circulation and impulse as the shock-generated flow, which has a non-uniform distribution of core vorticity. These quantities represent zeroth and first moments of the vorticity distribution, which cannot simultaneously be matched when a non-uniform distribution is approximated as a uniform one.

The impulse of the shock-generated flow is given by

$$
I\left(t=0^{+}\right) \equiv \int \rho \omega x \times \mathrm{d} \boldsymbol{A},
$$

so that

$$
I_{x}\left(t=0^{+}\right)=\int \rho \omega r \sin \theta \mathrm{d} A .
$$

This is similar to the integral for circulation derived earlier, except for the additional term $\rho r \sin \theta$. Following a similar argument, we find

$$
I_{x}\left(t=0^{+}\right)=\frac{\pi}{2} \frac{1}{V_{s}} \frac{\Delta p \Delta \rho}{\rho} R_{0}^{2} .
$$

Taking $\rho_{2}$ as the reference density and non-dimensionalizing gives

$$
\begin{aligned}
\bar{I}_{x}\left(\bar{t}=0^{+}\right) & =\frac{\pi}{2 \gamma_{1}}\left(\frac{1}{M}\right)\left(\frac{\bar{p}_{2}}{\bar{p}_{1}}-1\right)\left(\frac{\bar{\rho}_{1}}{\bar{\rho}_{2}}\right)\left(1-\frac{\bar{\rho}_{L}}{\rho_{H}}\right) \\
& \approx\left(\frac{1}{M}\right)\left(\frac{\bar{p}_{2}}{\bar{p}_{1}}-1\right)\left(\frac{\bar{\rho}_{1}}{\bar{\rho}_{2}}\right)\left(1-\frac{\bar{\rho}_{L}}{\bar{\rho}_{H}}\right) .
\end{aligned}
$$

Table 5 shows a comparison of the quantities $\bar{I}_{x}^{\prime}$ and $\bar{I}_{x}\left(\bar{l}=0^{+}\right)$for the canonical flow computations. Note that a value of $\frac{1}{2}$ has been assigned to $\bar{\rho}_{\text {model }}$ in order to collapse the data, and therefore, from this point on, $\bar{I}_{x}^{\prime} \equiv 0.5 \bar{y}_{\infty} \bar{\Gamma}$. The agreement is quite good. At this point, both $\bar{\Gamma}$ and $\bar{I}_{x}^{\prime}\left(=0.5 \bar{y}_{\infty} \bar{\Gamma}\right)$ have been successfully modelled as a function of $M$ and $\bar{\rho}_{L} / \bar{\rho}_{H}$. These results may be combined to give

$$
\bar{y}_{\infty}=\frac{\gamma_{1}}{2}\left(1+\frac{\bar{\rho}_{L}}{\bar{\rho}_{H}}\right) .
$$

Computed and predicted $\bar{y}_{\infty}$ data are also listed in table 5 . 


\begin{tabular}{lccccc} 
& & & & \multicolumn{2}{c}{$\bar{y}_{\infty}$} \\
\cline { 5 - 6 }$M$ & $\bar{\rho}_{L} / \bar{\rho}_{H}$ & $\bar{I}_{x}^{\prime}$ & $\bar{I}_{x}\left(\bar{t}=0^{+}\right)$ & Computed & Predicted \\
1.05 & 0.138 & 0.086 & 0.091 & 0.72 & 0.797 \\
1.1 & 0.138 & 0.169 & 0.164 & 0.75 & 0.797 \\
1.2 & 0.138 & 0.295 & 0.275 & 0.82 & 0.797 \\
1.5 & 0.138 & 0.485 & 0.450 & 1.00 & 0.797 \\
2.0 & 0.138 & 0.600 & 0.566 & 1.00 & 0.797 \\
1.1 & 0.354 & 0.112 & 0.123 & 0.82 & 0.948 \\
2.0 & 0.354 & 0.459 & 0.424 & 1.20 & 0.948 \\
2.0 & 0.569 & 0.269 & 0.283 & 1.20 & 0.948
\end{tabular}

TABLE 5. Computed and predicted impulse and spacing for the canonical flows

The computations show a dependence on both light/heavy gas density ratio and shock strength. The model predicts the density dependence to within about $20 \%$, but fails to predict the shock-strength dependence. The model was derived by combining the circulation and impulse models, and therefore suffers from the cumulative errors of both. Either model alone almost completely captures the dependence of $\bar{\Gamma}$ on $M$. Unfortunately, when taken together, the dependences combine in such a way as to completely cancel, when they should only partially cancel.

The expression for $\bar{y}_{\infty}$ completes the description of the vortex pair. The models for circulation, characteristic time, normalized velocity, and impulse predict the corresponding computed values quite well. Combining the strength and impulse models gives a model for spacing. This spacing model acceptably predicts the dependence on density ratio, but fails to predict the dependence on shock strength.

\section{Comparison with a three-dimensional steady flow}

Shock-induced vortical flows are conveniently studied in the two-dimensional unsteady case, while technological applications typically involve three-dimensional steady flows. As mentioned earlier, Marble has proposed an analogy between these two flows. We illustrate this analogy by comparison of a three-dimensional steady flow and its two-dimensional unsteady counterpart.

\subsection{Qualitative}

Waitz (1992) performed three-dimensional steady numerical simulations of contoured wall injectors for a SCRAMJET combustion chamber. Figure 13 shows one of his cases. A high-pressure helium jet (not shown) is injected horizontally outward from a downward-sloping rectangular nozzle. Supersonic air flows down the troughs on either side of the injector. An oblique shock (not shown) is formed by the intersection of the downward-sloping troughs and the horizontal combustor wall. The shock slices upward through the helium jet, depositing vorticity around the portion of its perimeter perpendicular to the shock. This forms a vortex pair which mixes the helium and air and lifts the jet away from the wall. The transverse edges of the graph are planes of symmetry. In addition to shock-generated vorticity, there is a contribution from shearinduced vorticity at the edges of the injector. This case, although not a purely shockgenerated vortical flow, affords the closest available comparison at the present time. Because both vortices are deposited at the same location, their overall effect should be 


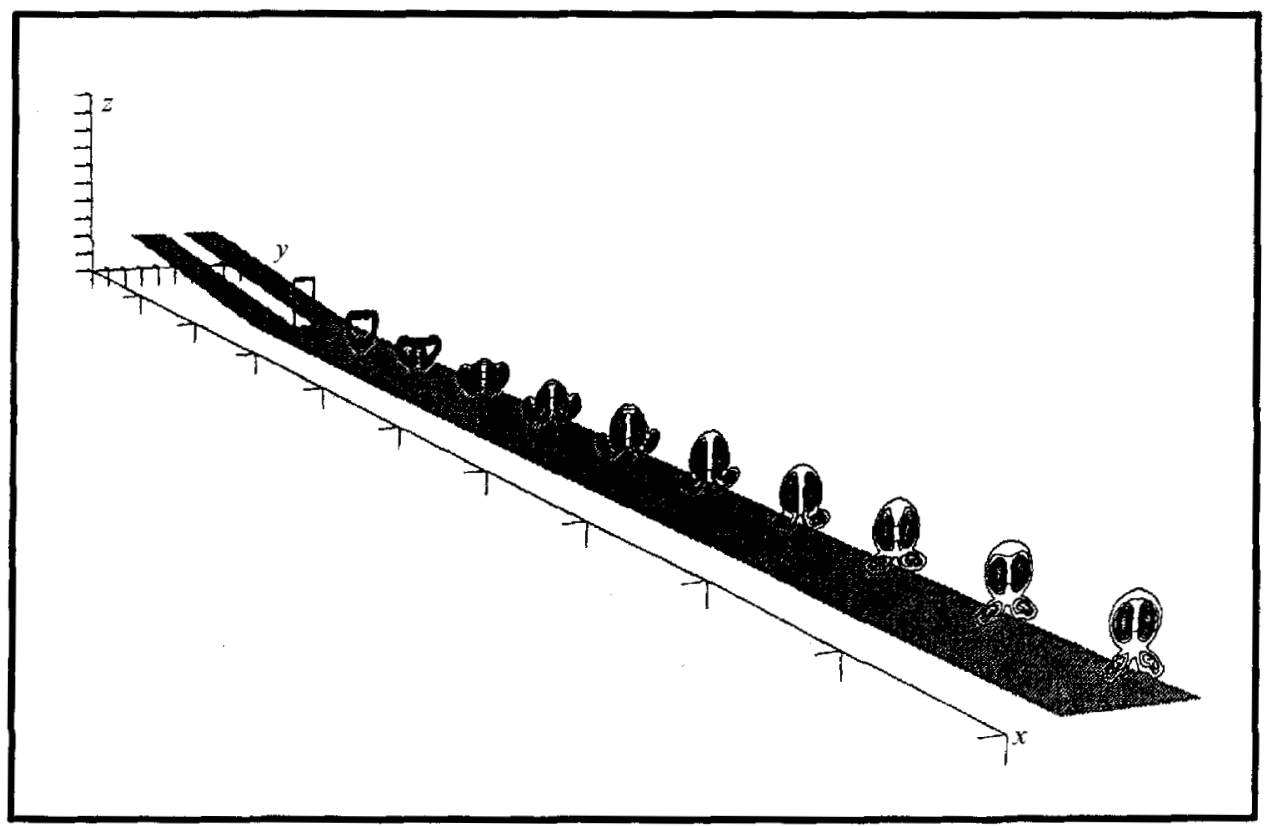

FIGURF 13. Waitz's computation of a three-dimensional steady shock-induced vortical flow (reproduction from Waitz 1992, p. 11).

similar to that of a purely shock-generated vorticity of magnitude equal to their combined strengths.

Figure 14 shows a two-dimensional unsteady purely shock-generated vortical flow closely corresponding to figure 13 . The vertical direction in the three-dimensional steady flow is analogous to time in the two-dimensional unsteady flow. The upper and lower walls of the two-dimensional flow correspond to the planes of symmetry in the three-dimensional flow, the left end of the two-dimensional flow is a wall corresponding to the horizontal combustor wall downstream of the injector, and the open right end of the two-dimensional flow corresponds to the open top of the injector. The height of the rectangular jet in the two-dimensional flow corresponds to the transverse width of the injector in the three-dimensional flow. The light/heavy gas density ratio and pressure and density jumps across the shocks are also matched: $\bar{\rho}_{L} / \bar{\rho}_{H}=0.082$, $\bar{p}_{2} / \bar{p}_{1}=1.949$, and $\bar{\rho}_{2} / \bar{\rho}_{1}=1.597$.

Visual comparison shows good agreement between the flows, especially up to time $\bar{t}=12$ in the two-dimensional case. This is evidence that the present studies, although more idealized than the flow in an actual injector, capture the main phenomena of interest. Beyond $\bar{t}=12$, small differences appear in the details of the flows, most notably at the downstream end of the two-dimensional structure and at the uppermost portion of the three-dimensional structure. This is most likely a consequence of greater dissipation in the three-dimensional flow. Nevertheless, the general features are still fairly well correlated.

\subsection{Quantitative}

Direct comparison of the two computed flows shows that their vortex pair strengths are similar. The circulation in figure 14 is 2.24 . The total circulation in figure 13 , using the same normalization, is 2.64 - a difference of less than $20 \%$. This limited difference, and their close qualitative agreement, suggest a close correspondence. We propose a mathematical description of Marble's analogy relating the temporal developments of 


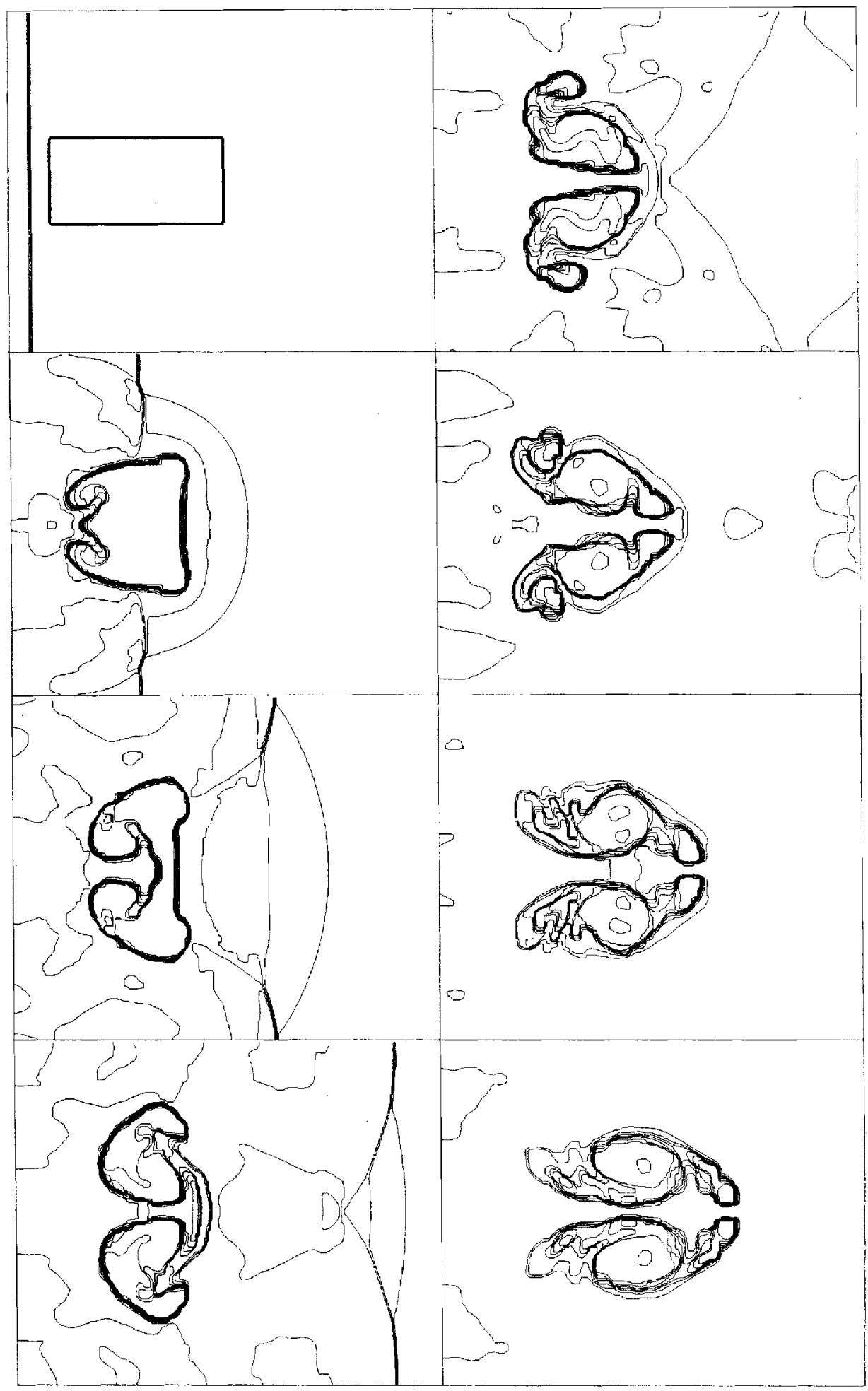

FIGURE 14. Density contours for a two-dimensional unsteady flow closely corresponding to figure 13 at times $\bar{t}=0,3,6,9,12,15,18,21$. Each plot contains nine equally spaced contours spanning the $10 \%$ to $90 \%$ levels between $\bar{\rho}_{\text {min }}$ and $\bar{\rho}_{m a x}$ in the instantaneous flow field. The minimum and maximum levels are, respectively, $\left(\bar{\rho}_{\min }, \bar{\rho}_{\max }\right)=(0.082,1.597),(0.101,1.964), \quad(0.116,1.908)$, $(0.116,1.997),(0.125,1.831),(0.137,1.747),(0.169,1.649)$, and $(0.177,1.615)$. 


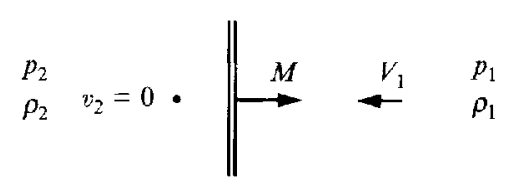

(a)

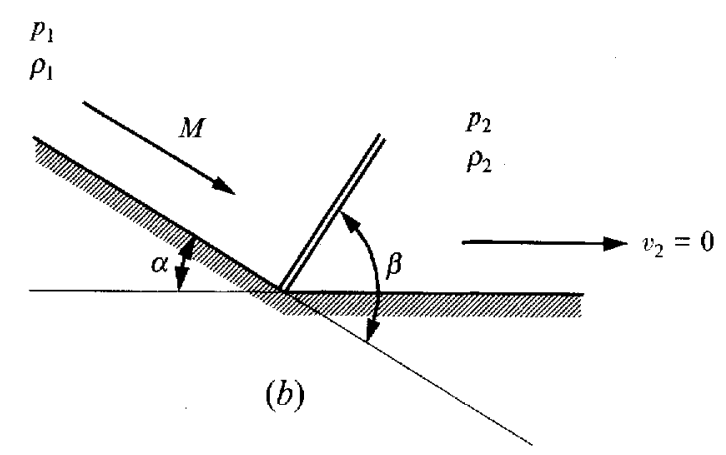

Figure 15. Analogy between two- and three-dimensional fows.

a two-dimensional unsteady vortical flow to the spatial development of a threedimensional steady vortical flow. The two computed flows provide a crude test of the proposed relationship; however, a definitive test is impossible owing to their inexact correspondence.

Two- and three-dimensional shock waves are shown schematically in figure $15(a, b)$. The pressure and density jumps across the shock are:

two-dimensional

$$
\begin{aligned}
& \frac{p_{2}}{p_{1}}=1+\frac{2 \gamma_{1}}{\gamma_{1}+1}\left(M_{2 D}^{2}-1\right), \\
& \frac{\rho_{2}}{\rho_{1}}=\frac{\left(\gamma_{1}+1\right) M_{2 D}^{2}}{\left(\gamma_{1}-1\right) M_{2 D}^{2}+2}
\end{aligned}
$$

three-dimensional

$$
\begin{aligned}
M_{3 D}^{2} & =\frac{2 \cos (\beta-\delta)}{(\sin \beta)\left[\sin (2 \beta-\delta)-\gamma_{1} \sin \delta\right]}, \\
\frac{p_{2}}{p_{1}} & =1+\frac{2 \gamma_{1}}{\gamma_{1}+1}\left(M_{3 D}^{2} \sin ^{2} \beta-1\right), \\
\frac{\rho_{2}}{\rho_{1}} & =\frac{\left(\gamma_{1}+1\right) M_{3 D}^{2} \sin ^{2} \beta}{\left(\gamma_{1}-1\right) M_{3 D}^{2} \sin ^{2} \beta+2} .
\end{aligned}
$$

Correspondence of the pressure and density jumps requires that $M_{2 D}=M_{3 D} \sin \beta$, where $\beta$ is determined from $M_{3 D}$ and the turning angle $\delta$. The velocity $(\mathrm{d} \bar{x} / \mathrm{d} \bar{t})_{2 D}$ may be related to a corresponding slope $(\mathrm{d} \bar{z} / \mathrm{d} \bar{x})_{3 D}$ by

$$
(\mathrm{d} \bar{x} / \mathrm{d} \bar{t})_{2 D}=m(\mathrm{~d} \bar{z} / \mathrm{d} \bar{x})_{3 D}
$$

where $m$ is determined by matching the shock motion:

$$
M_{2 D}=m \tan (\beta-\delta) \text {. }
$$

For the two- and three-dimensional computations shown earlier, $M_{3 D}=6$ and 
$\delta=\tan ^{-1} \frac{1}{12}$, so that $\beta=0.2263 \mathrm{rad}, M_{2 D}=1.346$, and $m=9.34$. We thus compare the two-dimensional trajectory of the centre of mass fraction and the three-dimensional jet lift-off:

and

$$
\begin{gathered}
(\mathrm{d} \bar{x} / \mathrm{d} \bar{t})_{2 D}=0.18 \\
m(\mathrm{~d} \bar{z} / \mathrm{d} \bar{x})_{3 D}=(9.34)(0.017)=0.16 .
\end{gathered}
$$

The agreement is better than expected, considering the $20 \%$ difference in vortex pair strengths. This suggests a trajectory correspondence governed by the matching of geometry, light/heavy gas density ratio, shock pressure, and density jumps. Together with the observed qualitative agreement, this quantifies Marble's idea that the twodimensional unsteady flow can be directly associated with a corresponding threedimensional steady flow. Rigorous verification awaits a comparison of exactly corresponding cases.

\section{Conclusions}

The canonical shock-induced vortical flow is the two-dimensional unsteady passage of a shock wave over a single circular inhomogeneity ('jet') of light gas. The shock wave provided an upstream-pointing pressure gradient, and the light/heavy interface provided a radially outward density gradient. The interaction of these gradients generated vorticity in accordance with the vorticity production term of the vorticity equation

$$
\frac{\mathrm{D} \omega}{\mathrm{D} t}=\frac{1}{\rho^{2}}(\nabla \rho \times \nabla p)+\ldots
$$

This vorticity caused the jet to roll up into a kidney shape, counterclockwise in the upper half-plane and clockwise in the lower half-plane. As time went on, the structure evolved towards a vortex pair of finite core size. This vortex pair moved downstream relative to the ambient fluid due to the motion each vortex induced in the other. The velocity and circulation of this vortex pair were essentially constant throughout the evolution of the flow. Together with the late-time core spacing, these variables constituted a complete fluid-mechanical description of the flow.

The governing equations were integrated for various initial conditions, and the above flow variables were tabulated from these computations. Algebraic models were derived for the late-time circulation and spacing, and characteristic time of development. These models were successfully used to correlate the computational results, except for the spacing model, which failed to capture the dependence on Mach number. In addition, a perturbation analysis was performed to describe the dependence of the normalized velocity of the vortex pair on its two geometrical parameters: the ratio of vortex size to vortex spacing, and the ratio of vortex spacing to channel spacing. This model gave good agreement with the computations for a wide range of different initial conditions.

All of the above results were for studies of two-dimensional unsteady flows, while real technological applications are more likely to be three-dimensional and steady. Marble proposed an analogy between spatial development in the three-dimensional steady flow and temporal development in the two-dimensional unsteady flow that can be used to relate the two flows. Good qualitative agreement was found between a twodimensional unsteady flow and a similar, but not exactly corresponding, threedimensional steady flow. A mathematical description of Marble's analogy was proposed, and quantitative comparison of the two flows supported the analogy. 
This work was supported by the Air Force Office of Scientific Research through contract number F49620-86-C-0113 and grant number AFOSR-90-0188, under the supervision of Dr Julian Tishkoff, and by the National Science Foundation through a Cray supercomputer grant at the San Diego Supercomputer Center. The first author was supported by the Office of Naval Research through an ONR Graduate Fellowship. The authors would like to thank Dr Elaine S. Oran, of the Laboratory for Computational Physics at the Naval Research Laboratory for providing the algorithm used in the numerical simulations.

\section{REFERENCES}

Boris, J. P. \& BoOK, D. L. 1973 Flux-corrected transport. I. SHASTA, a fluid transport algorithm that works. J. Comput. Phys. 11, 38-69.

HAAS, J.-F. 1984 Interaction of wcak shock waves and discrete gas inhomogeneities. $\mathrm{PhD}$ thesis. California Institute of Technology.

HaAs, J.-F. \& Sturtivant, B. 1987 Interaction of weak shock waves with cylindrical and spherical gas inhomogeneities. J. Fluid Mech. 181, 41-76.

HAWLEY, J. F. \& ZABUSKY, N. J. 1989 Vortex paradigm for shock-accelerated density interfaces. Phys. Rev. Lett. 63, 1241-1244.

Hendricks, G. J. \& Marble, F. E. 1991 Shock enhancement of supersonic combustion processes. In preparation.

JACOBS, J. W. 1992 Shock-induced mixing of a light-gas cylinder. J. Fluid Mech. 234, 629-649.

Marble, F. E., Hendricks, G. J. \& Zukoski, E. E. 1987 Progress toward shock enhancement of supersonic combustion processes. AIAA Paper 87-1880.

Marblf, F. E., Zukoski, E. E., Jacobs, J. W., Hendricks, G. J. \& Waitz, I. A. 1990 Shock enhancement and control of hypersonic mixing and combustion. AIAA Paper 90-1981.

Moore, D. W. \& Pullin, D. I. 1987 The compressible vortex pair. J. Fluid Mech. 185, 171-204.

ORAN, E.S. 1991 LCPFCT - A monotone algorithm for solving continuity equations. In preparation.

PICONe, J. M. \& Boris, J. P. 1988 Vorticity generation by shock propagation through bubbles in a gas. J. Fluid Mech. 189, 23-51.

Picone, J. M., Oran, E. S., Boris, J. P. \& Young, T. R. 1985 Theory of vorticity generation by shock wave and flame interactions. In Dynamics of Shock Waves, Explosions, and Detonations, pp. 429-448. AIAA.

Pierrehumbert, R. T. 1980 A family of steady, translating vortex pairs with distributed vorticity. J. Fluid Mech. 99, 129-144.

Rudinger, G. \& Somers, L. M. 1960 Behaviour of small regions of different gases carried in accelerated gas flows. J. Fluid Mech. 7, 161-176.

WaITZ, I. A. 1992 Vorticity generation by contoured wall injectors. AIA A Paper 92-3550.

YANG, J. \& KubotA, T. 1993 The steady motion of a symmetric, finite core size, counterrotating vortex pair in an unbounded domain. SIAM J. Appl. Maths (to appear).

YANG, J., KubOTA, T. \& ZUKOSKI, E. E. 1993 Applications of shock-induced mixing to supersonic combustion. AIAA J. 31, 854-862. 